\title{
Rate-Splitting Multiple Access for Multi-antenna Downlink Communication Systems: Spectral and Energy Efficiency Tradeoff
}

\author{
Gui Zhou, Yijie Mao, Member, IEEE, Bruno Clerckx, Senior Member, IEEE
}

\begin{abstract}
Rate-splitting (RS) has recently been recognized as a promising physical-layer technique for multi-antenna broadcast channels (BC). Due to its ability to partially decode the interference and partially treat the remaining interference as noise, RS is an enabler for a powerful multiple access, namely rate-splitting multiple access (RSMA), that has been shown to achieve higher spectral efficiency (SE) and energy efficiency (EE) than both space division multiple access (SDMA) and non-orthogonal multiple access (NOMA) in a wide range of user deployments and network loads. As SE maximization and EE maximization are two conflicting objectives in the moderate and high signal-to-noise ratio (SNR) regimes, the study of the tradeoff between the two criteria is of particular interest. In this work, we address the SE-EE tradeoff by studying the joint SE and EE maximization problem of RSMA in multiple input single output (MISO) BC with rate-dependent circuit power consumption at the transmitter. To tackle the challenges coming from multiple objective functions and rate-dependent circuit power consumption, we first propose two models to transform the original problem into two single-objective problems, namely, weighted-sum method and weighted-power method. A low-complexity algorithm with closed-form solution is proposed to solve each single-objective problem in the two-user system. For the generalized $K$-user system, a successive convex approximation (SCA)-based algorithm is then proposed to optimize the precoders of each transformed problem. Numerical results show that our algorithm converges much faster than existing algorithms. In addition, the performance of RSMA is superior to or equal to SDMA and NOMA in terms of both SE and EE and their tradeoff.
\end{abstract}

Index Terms-Rate-splitting, spectral efficiency, energy efficiency, precoder design, successive convex approximation (SCA).

\section{INTRODUCTION}

Rate-splitting (RS) has recently emerged in multi-antenna broadcast channel (BC) as a powerful and robust nonorthogonal transmission technique and interference management strategy for cellular networks. At the base station (BS), the message intended for each user is split into a common and a private part. After jointly encoding the common parts into a common stream to be decoded by all users and independently encoding the private parts into the private streams for the corresponding users only, BS

G. Zhou is with the School of Electronic Engineering and Computer Science at Queen Mary University of London, London E1 4NS, U.K. (email: g.zhou@qmul.ac.uk). B. Clerckx and Y. Mao are with the Electrical and Electronic Engineering Department, Imperial College London, London SW7 2AZ, U.K. (e-mail: b.clerckx, y.mao16@imperial.ac.uk). linearly precodes all the encoded streams and broadcasts the superimposed data streams to all users. By allowing each user to sequentially decode the common stream and the intended private stream with the assistance of successive interference cancellation (SIC), RS grants all users the ability to partially decode the interference and partially treat the remaining interference as noise.

The study of RS dates back to early 1980s in [1] for the two-user single-input single-output (SISO) interference channel (IC). The flexibility of RS in dealing with interference in SISO IC has motivated the investigation of the benefits of RS in modern multiple input single output (MISO) BC [2], [3]. As the two fundamental indicators for a communication system design, spectral efficiency (SE) demonstrates the amount of information to be transmitted per unit of time while energy efficiency (EE) demonstrates how much information rate can be transmitted per unit of energy. Existing literature of RS in MISO BC has shown that $\mathrm{RS}$ is an enabler for a powerful multiple access, namely ratesplitting multiple access (RSMA), that achieves both higher $\mathrm{SE}$ and EE over space division multiple access (SDMA) and non-orthogonal multiple access (NOMA) in both perfect Channel State Information at the Transmitter (CSIT) [4]- [7] and imperfect CSIT [8]-[14]. Compared with RSMA that dynamically partially decodes the interference and partially treats interference as noise, SDMA and NOMA fall into two extreme interference management cases where users in SDMA always decode their intended signal by fully treating any residual interference as noise and users in NOMA always fully decode the interference generated by the users with weaker channel strengths [5]. Besides conventional MISO BC, the benefits of RSMA in the SE domain have been further demonstrated in massive MISO system [9], millimeter wave system [10], overloaded system [13], MISO $\mathrm{BC}$ with user relaying [15], [16], etc. The benefits of RSMA from an EE perspective are also investigated in MISO BC [17], and MISO BC with a common message (so-called nonorthogonal unicast and multicast transmission) [4].

The results in the existing literature [18], [19] clarify that both EE and SE increase with the signal-to-noise ratio (SNR) in the low SNR regime. Therefore, both EE and SE are maximized by consuming all transmit power. However, in the moderate and high SNR regimes, SE still increases with SNR while EE starts decreasing with SNR. In another words, 
SE is maximized by making use of the full available transmit power, while this is not the case for EE where transmitting using a portion of the available transmit power budget is preferred to maximize EE. Therefore, EE and SE conflict with each other in the moderate and high SNR regimes leading to a fundamental tradeoff between SE and EE.

In recent years, the existence of the SE-EE tradeoff is well recognized in the literature, as shown in [20]-[22]. In some cases, the tradeoff is achieved by optimizing the EE metric constrained by the SE and transmit power constraints [20]. The higher the SE target, the lower the EE generated. However, such method requires a given SE constraint and the optimization problem comes with a feasibility issue. Whether the given SE constraint can be met requires extra feasibility testing. Jointly optimizing SE and EE gets rid of those issues and it is a more flexible and general method to deal with the SE-EE tradeoff problem [21], [22].

The SE-EE tradeoff optimization problem is a multiobjective optimization (MOO) problem which is generally transformed to its corresponding single-objective optimization (SOO) problem by adopting the weighted sum method [21], [23]. Two widely used precoder design frameworks to solve the transformed SOO problem are Dinkelbach's framework [24] [19] and successive convex approximation (SCA) framework [25] [26] or called inner approximation (IA) framework [27]. The key step of the Dinkelbach's framework is to transform the fractional program into a sequence of parametric problems by introducing an auxiliary variable, which can then be solved by zero forcing (ZF) [28], weighted minimum mean square error (WMMSE) [29], SCA methods [30], monotonic optimization [31], [32]. It is indeed a two-layer iterative algorithm framework, which optimizes the parameter in the outer layer and precoder in the inner layer. In comparison, SCA framework can be directly applied to solve the SE-EE tradeoff problem by approximating the fractional EE metric as well as other non-convex expressions into their convex approximation counterparts, which results in a one-layer iterative algorithm. The SCA framework has shown its performance advantages in terms of convergence compared to the Dinkelbach's framework in conventional communication systems with orthogonal multiple access (OMA) [26].

In this work, we investigate the SE-EE tradeoff of RSMA in multi-user multi-antenna systems. The major contributions of this work are as follows:

- We investigate the SE-EE tradeoff achieved by RSMA in a MISO BC. Previous works on RSMA either address the SE (as in [4]-[13]) or EE (as in [4], [17]), but the tradeoff between SE and EE has never been studied. It should be reminded that, since RSMA is a general framework for non-orthogonal transmission that subsumes SDMA, NOMA, OMA and multicasting as special cases [3], [5], identifying the SE-EE tradeoff of RSMA automatically solves the SE-EE tradeoff of those particular strategies.

- We formulate a MOO problem that jointly maximizes
SE and EE of RSMA in a MISO BC subject to a transmit power constraint. To obtain reasonable operating points on the Pareto boundary that balance SE and EE, we adopt two different approaches to convert the MOO problem into a SOO problem, namely the weightedsum and the weighted-power approaches. The former transformation is achieved by maximizing the weighted sum of SE and EE and the latter is to minimize the weighted sum of the inverse of both SE and EE.

- To better characterize the SNR regime where SE and EE have conflicting tendencies as SNR increases, we first propose a low-complexity linear precoding algorithm for the two-user case and analytically study the optimal transmit power to maximize the SE and EE tradeoff. Motivated by the insights obtained from the two-user case, we investigate the optimal precoding method to maximize the SE-EE tradeoff in the generalized $K$-user case. Due to the non-convexity of the transformed SOO problems, we propose a SCA-based framework to solve both weighted-sum and weighted-power problems with two different rate lower bounds to relax the non-convex rate constraints, namely "LB I" and "LB II". LB I is achieved by exploiting the convexity of log function and directly using the first-order Taylor approximation of the rate function. In comparison, LB II is obtained by approximating the fractional signal-to-interferenceplus-noise ratio (SINR) expression only. The proposed SCA-based algorithm is shown to not only solve the SE-EE tradeoff problem but also the individual SE and EE problems.

- We demonstrate through numerical results that RSMA achieves a larger achievable SE and EE tradeoff regime than the conventional multi-user SDMA and NOMA in different user deployments, SE and EE weights, transmit power constraints, etc. The proposed lowcomplexity precoding algorithm, though with a much lower computational complexity, achieves almost the same trade-off performance as the proposed SCA suboptimal precoding algorithm. We further evaluate the convergence and complexity of the developed algorithm by observing the CPU time and the number of required iterations. The proposed SCA-based algorithm with both lower bound LB I and II converges within a few iterations and is faster than the existing Dinkelbach's algorithm. LB I converges slightly slower than LB II but both converge to similar boundary point. We conclude that RSMA can not only improve individual SE and EE, but also achieve a better SE-EE tradeoff in multiantenna BC.

The remaining of this paper is organized as follows. In Section II, the system model is specified and the optimization problems are formulated. The weighted-sum approach is proposed to design the precoder in Section III. A weightedpower approach is proposed to design the precoder in Section IV. Section V and Section VI show the numerical results and conclusion, respectively. 
Notations: Vectors and matrices are denoted by boldface lowercase and uppercase letters, respectively. The symbols $\mathbf{x}^{*}, \mathbf{x}^{\mathrm{T}}$ and $\mathrm{x}^{\mathrm{H}}$ denote the conjugate, transpose, and Hermitian (conjugate transpose) of vector $\mathbf{x}$, respectively. Additionally, the symbol $\mathbb{C}$ denotes complex field and $\mathbb{R}$ represents real field. The symbol $\|\mathbf{x}\|_{2}$ denotes 2-norm of vector $\mathbf{x}$ and the symbol $\|\mathbf{X}\|_{F}$ denotes Frobenius norm of matrix $\mathbf{X}$. The symbols $\operatorname{Tr}\{\cdot\}, \operatorname{Re}\{\cdot\}$, and $|\cdot|$ denote the trace, real part and modulus, respectively.

\section{SySTEM MOdEL AND PROBLEM FORMULATION}

In this section, we specify the system model of RSMA in MISO BC followed by the formulated SE and EE tradeoff problem. The rate-dependent power consumption model of the transmitter is also specified in this section.

\section{A. Rate-Splitting Transmit Signal Model}

In this work, we consider a downlink multi-antenna multiuser communication system where one BS with $N_{t}$ transmit antennas transmits $K$ messages simultaneously to $K$ singleantenna users. The principle of RSMA is shown in Fig. 11 In particular, at the BS, the message $W_{k}$ intended for user $k$ is split into a private part $W_{\mathrm{p}, k}$ and a common part $W_{\mathrm{c}, k}$. The private parts $W_{\mathrm{p}, 1}, \cdots, W_{\mathrm{p}, K}$ are independently encoded into the private streams $s_{1}, \cdots, s_{K}$ and the common parts of all users $W_{\mathrm{c}, 1}, \cdots, W_{\mathrm{c}, K}$ are combined into a common message $W_{\mathrm{c}}$, which is encoded into a common stream $s_{\mathrm{c}}$ using a public codebook. Denoting $\mathbf{s}=\left[s_{\mathrm{c}}, s_{1}, \cdots, s_{K}\right]^{\mathrm{T}}$ and assuming Gaussian signaling with $\mathbb{E}\left[\mathbf{s s}^{\mathrm{H}}\right]=\mathbf{I}$, the $K+1$ streams are linearly precoded by precoding vectors $\mathbf{f}_{\mathrm{c}}, \mathbf{f}_{1}, \cdots, \mathbf{f}_{K}$. Define set $\mathcal{K}=\{1,2, \ldots, K\}$ and set $\mathcal{K}_{\mathrm{c}}=\mathcal{K} \cup\{\mathrm{c}\}$, the resulting transmit signal of RSMA can be written as

$$
\mathbf{x}=\sum_{i \in \mathcal{K}_{\mathrm{c}}} \mathbf{f}_{i} s_{i}
$$

We use a compact notation $\mathbf{F}$ to denote the family of the precoder vectors as $\mathbf{F}=\left[\mathbf{f}_{\mathrm{c}}, \mathbf{f}_{1}, \cdots, \mathbf{f}_{K}\right] \in \mathbb{C}^{N_{\mathrm{t}} \times(K+1)}$. It belongs to the power constraint set $\mathcal{S}=\left\{\mathbf{F} \mid\|\mathbf{F}\|_{F}^{2} \leq \mathrm{P}_{\max }\right\}$ where $\mathrm{P}_{\max }$ is the transmit power limit at the BS.

The received signal of user $k$ is

$$
y_{k}=\mathbf{h}_{k}^{\mathrm{H}} \sum_{i \in \mathcal{K}_{\mathrm{c}}} \mathbf{f}_{i} s_{i}+n_{k},
$$

where $\mathbf{h}_{k} \in \mathbb{C}^{N_{\mathrm{t}} \times 1}$ is the channel between BS and user $k$ and noise $n_{k}$ follows Gaussian distribution, i.e., $\mathcal{N}\left(0, \sigma_{k}^{2}\right)$. In this work, we assume perfect CSITI.

At the receivers, each user firstly decodes the common stream by treating all the private streams as noise, then SIC is used to remove the decoded common stream from the

\footnotetext{
${ }^{1}$ The investigation of the SE-EE tradeoff in RSMA under imperfect CSIT will be considered in our future work.
}

received signal under the assumption of error-free decoding 2 . Each user then decodes its own private stream by treating other private streams as noise. Thus, the spectral efficiencies (bit/s/Hz) of the intended common and private streams at user $k$ are formulated as

$$
\begin{gathered}
R_{\mathrm{c}, k}(\mathbf{F})=\log _{2}\left(1+\frac{\mathbf{h}_{k}^{\mathrm{H}} \mathbf{f}_{\mathrm{c}} \mathbf{f}_{\mathrm{c}}^{\mathrm{H}} \mathbf{h}_{k}}{\sigma_{k}^{2}+\sum_{i \in \mathcal{K}} \mathbf{h}_{k}^{\mathrm{H}} \mathbf{f}_{i} \mathbf{f}_{i}^{\mathrm{H}} \mathbf{h}_{k}}\right), \\
R_{k}(\mathbf{F})=\log _{2}\left(1+\frac{\mathbf{h}_{k}^{\mathrm{H}} \mathbf{f}_{k} \mathbf{f}_{k}^{\mathrm{H}} \mathbf{h}_{k}}{\sigma_{k}^{2}+\sum_{i \in \mathcal{K} \backslash\{k\}} \mathbf{h}_{k}^{\mathrm{H}} \mathbf{f}_{i} \mathbf{f}_{i}^{\mathrm{H}} \mathbf{h}_{k}}\right) .
\end{gathered}
$$

The achievable rate of the common stream is given as $R_{\mathrm{c}}(\mathbf{F})=\min _{k \in \mathcal{K}} R_{\mathrm{c}, k}(\mathbf{F})$ to guarantee that all users are capable of decoding the common stream successfully.

Finally, the system SE is the sum of the SE of the common and private streams, which is given by

$$
f(\mathbf{F})=\sum_{k \in \mathcal{K}_{\mathrm{c}}} R_{k}(\mathbf{F}) .
$$

Remark 1: Please keep in mind that the contribution of the common stream is observed by comparing RSMA and traditional multiple access techniques of SDMA and NOMA. Since this work considers sum SE, we can see from (5) that splitting only one user's message or multiple users' messages does not change the problem formulation as well as its performance. Therefore, the split of a single user's message is sufficient in this work.

\section{B. Spectral Efficiency Maximization}

The existing literature [5], [9]-[13], [15] have investigated precoder design of RSMA for maximizing the SE in various scenarios. The SE maximization problem is formulated as follows

$$
\max _{\mathbf{F} \in \mathcal{S}} f(\mathbf{F}) .
$$

Popular precoding techniques rely on closed form precoders based on ZF method [28] for private messages as in [3], [8]-[10], or optimized precoders based on convex optimization commonly relying on an extended version of the WMMSE method [29], as in [5], [11]-[13], [15]).

\section{Energy Efficiency Maximization}

As another important performance metric, EE benefits of RSMA in multi-antenna BC have only been studied in a few literature [4], [17] and only constant circuit power consumption is considered in those works. However, in practical communication systems, the circuit power consumption contains two parts, namely the rate-independent

\footnotetext{
${ }^{2}$ In this paper we use information theoretical rate expressions. It means that the codeword lengths are long enough to apply Shannon theoretical arguments and there is no decoding error. Since the obtained rates are achievable, there is no error in decoding, and in the successive interference cancellation process. In other words, perfect SIC is not assumed for simplicity, but is a result of good channel codes. The impact of finite block length and imperfect SIC on the system performance is beyond the scope of this work, which will be our future work.
} 


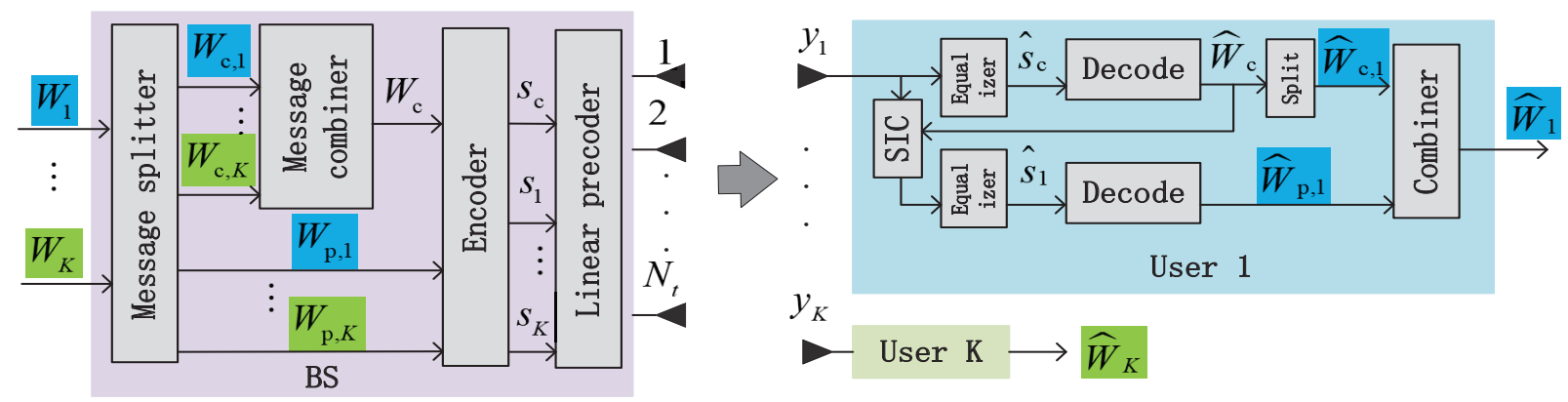

Fig. 1: System architecture of RSMA for MISO BC.

fixed part and the rate-dependent dynamic part [33], [34]. The former is for basic circuit operations, e.g., channel estimation, precoder chains, and linear processing at the BS while the latter is for information processing, e.g., coding, decoding and backhaul power consumption. In this work, we consider a more practical power consumption model that has not been studied in the literature of RSMA yet. We adopt the power consumption model of [34], [35], where the ratedependent circuit power consumption is written as a linear function of the system sum-rate. Therefore, the total power consumption is modeled as

$$
g(\mathbf{F})=\|\mathbf{F}\|_{F}^{2}+\mathrm{P}_{\mathrm{c}}+\chi f(\mathbf{F}),
$$

where $\|\mathbf{F}\|_{F}^{2}$ is the transmit power consumption, $\mathrm{P}_{\mathrm{c}}$ is a constant representing the rate-independent fixed power consumption, and $\chi \geq 0$ is a constant demonstrating the coding, decoding, and backhaul power consumption per unit data rate $(\mathrm{W} /(\mathrm{bit} / \mathrm{s} / \mathrm{Hz}))$ [33].

The corresponding EE maximization problem under the practical power consumption model is formulated as

$$
\max _{\mathbf{F} \in \mathcal{S}} \frac{f(\mathbf{F})}{g(\mathbf{F})} .
$$

Problem (8) is a fractional programming with non-concave numerator and non-convex denominator, which is hard to be solved directly. In addition, the non-convex practical power consumption model with non-linear rate expression makes the entire problem more complex to solve.

\section{Spectral Efficiency and Energy Efficiency Tradeoff}

The system SE is an increasing function of the transmit power consumption and its maximization is achieved by consuming all available transmit power. Such a strategy may not be suitable for EE maximization, since EE tries to balance SE and power consumption. Hence, EE and SE are two conflicting metrics in the moderate and high SNR regimes, which results in a SE-EE tradeoff. In this subsection, we characterize this tradeoff and identify the precoder strategy that achieves the best SE-EE tradeoff. The SE-EE tradeoff is an MOO problem, which is given by

$$
\max _{\mathbf{F} \in \mathcal{S}}\left[\frac{f(\mathbf{F})}{g(\mathbf{F})}, f(\mathbf{F})\right] .
$$

Note that the SE maximization Problem (6) and the EE maximization Problem (8) are actually special cases of the SE-EE tradeoff Problem (9). As a consequence, individual problems (6) and (8) can also be solved under the framework of our proposed algorithm for Problem (9).

In the following, we solve the MOO Problem (9) by two methods. In particular, we first directly adopt the weightedsum method to obtain a weakly Pareto optimal solution 3 of Problem (9) in Section III. In Section IV, inspired by the weighted-sum method, a weighted-power method is developed to characterize the SE-EE tradeoff, which is demonstrated to have the same performance as the weightedsum method in the numerical results section.

\section{WEIGHED-SUM APPROACH}

In this section, the weighted-sum approach to solve Problem (9) is specified. Weighted-sum approach is a classical method to solve MOO. It converts MOO into a SOO problem by assigning a weight to each normalized objective function and sum them up. Therefore, the MOO of 9 is transformed into

$$
\max _{\mathbf{F} \in \mathcal{S}} w \frac{f(\mathbf{F})}{g(\mathbf{F})}+(1-w) \frac{f(\mathbf{F})}{\mathrm{P}_{\mathrm{c}}},
$$

where $w \in[0,1]$ is a constant to characterize the priorities of EE and SE and is decided according to the application environments and quality of service (QoS) requirements. As specified in (7), $\mathrm{P}_{\mathrm{c}}$ is a constant representing the rateindependent fixed power consumption. According to [23], the denominator $\mathrm{P}_{\mathrm{c}}$ of the second fraction in (10) is introduced to unify the units of the two objective functions in (9) so that the values are comparable. We remark that the denominator of the second term is a constant which could be chosen randomly without affecting the solutions of Problem (10).

\footnotetext{
${ }^{3}$ According to Proposition 3.9 in [36], the optimal solution of the corresponding weighted-sum problem is weakly Pareto optimal for the original MOO problem.
} 


\section{A. Low-Complexity Algorithm for Two-User System}

In this section, a low-complexity algorithm with closedform solution is developed for a two-user system by extending the SE analysis of [37] to the SE-EE tradeoff. Based on the proposed low-complexity algorithm, we gain important insights on the EE-SE trade-off problem.

To that end, the precoding vectors for the private streams are defined as $\mathbf{f}_{k}=\sqrt{p_{k}} \mathbf{v}_{k}, k=1,2$, where $p_{k}$ is the transmit power allocated to the $k$-th private stream, and $\mathbf{v}_{k}$ is the precoding direction which is fixed by using $\mathrm{ZF}$

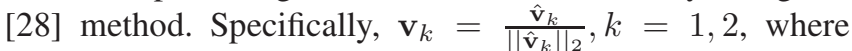
$\left[\hat{\mathbf{v}}_{1}, \hat{\mathbf{v}}_{2}\right]=\mathbf{H}\left(\mathbf{H}^{\mathrm{H}} \mathbf{H}\right)^{-1}$ and $\mathbf{H}=\left[\mathbf{h}_{1}, \mathbf{h}_{2}\right]$. Thus, we have $\left|\mathbf{h}_{2}^{\mathrm{H}} \mathbf{f}_{1}\right|=0,\left|\mathbf{h}_{1}^{\mathrm{H}} \mathbf{f}_{2}\right|=0$, and $\left|\mathbf{h}_{k}^{\mathrm{H}} \mathbf{f}_{k}\right|^{2}=\rho p_{k}|| \mathbf{h}_{k} \|^{2}, k=1,2$, where $\rho=1-\left|\overline{\mathbf{h}}_{1}^{\mathrm{H}} \overline{\mathbf{h}}_{2}\right|^{2}$ and $\overline{\mathbf{h}}_{k}=\frac{\mathbf{h}_{k}}{\left.\mathbf{h}_{k}\right|_{2}}, k=1,2$. Furthermore, assume that the sum rate of the SE-EE tradeoff problem in (10) is maximized by using transmit power $\|\mathbf{F}\|_{F}^{2}=p_{x}$ (where $p_{x} \leq \mathrm{P}_{\max }$ ), where the private streams are allocated with transmit power $p_{1}, p_{2}$ such that $p_{1}+p_{2}=$ $t p_{x}$ with $0 \leq t \leq 1$, and the common stream is assigned with transmit power $(1-t) p_{x}$. With fixed $t$ and $p_{x}$, the optimal power allocation for the private streams to maximize the sum rate is the Water-Filling solution, which is given by

$$
\begin{aligned}
& p_{1}=\max \left(\mu\left(t, p_{x}\right)-\frac{1}{\beta_{1}}, 0\right), \\
& p_{2}=\max \left(\mu\left(t, p_{x}\right)-\frac{1}{\beta_{2}}, 0\right),
\end{aligned}
$$

where $\beta_{1}=\frac{\rho\left\|\mathbf{h}_{1}\right\|^{2}}{\sigma_{1}^{2}}$ and $\beta_{2}=\frac{\rho\left\|\mathbf{h}_{2}\right\|^{2}}{\sigma_{2}^{2}}$. The water level $\mu\left(t, p_{x}\right)$ is chosen such that $p_{1}+p_{2}=t p_{x}$, and set as $\mu\left(t, p_{x}\right)=\frac{t p_{x}}{2}+\frac{1}{2 \beta_{1}}+\frac{1}{2 \beta_{2}}$. by

The precoding vector for the common stream is designed

$$
\begin{aligned}
& \max _{\mathbf{f}_{\mathrm{c}}} \min \left(\frac{\left|\mathbf{h}_{1}^{\mathrm{H}} \mathbf{f}_{\mathrm{c}}\right|^{2}}{\sigma_{1}^{2}+\left|\mathbf{h}_{1}^{\mathrm{H}} \mathbf{f}_{1}\right|^{2}}, \frac{\left|\mathbf{h}_{2}^{\mathrm{H}} \mathbf{f}_{\mathrm{c}}\right|^{2}}{\sigma_{2}^{2}+\left|\mathbf{h}_{2}^{\mathrm{H}} \mathbf{f}_{2}\right|^{2}}\right) \\
& \text { s.t. } \|\left.\mathbf{f}_{\mathrm{c}}\right|^{2}=(1-t) p_{x} .
\end{aligned}
$$

According to [37], when $p_{1}>0$ and $p_{2}>0$, Problem (12) is equivalent to

$$
\max _{\mathbf{f}_{\mathrm{c}}} \min \left(\left|\overline{\mathbf{h}}_{1}^{\mathrm{H}} \mathbf{f}_{\mathrm{c}}\right|^{2},\left|\overline{\mathbf{h}}_{2}^{\mathrm{H}} \mathbf{f}_{\mathrm{c}}\right|^{2}\right) \text {, s.t. }\left\|\mathbf{f}_{\mathrm{c}}\right\|^{2}=(1-t) p_{x} .
$$

See [37] for more details about the equivalence of Problems (12) and (13). Then, it follows from [38] that the optimal $\mathbf{f}_{\mathrm{c}}$ satisfies the equations $\frac{\left|\mathbf{h}_{1}^{\mathrm{H}} \mathbf{f}_{\mathrm{c}}\right|^{2}}{\sigma_{1}^{2}+\left|\mathbf{h}_{1}^{\mathrm{H}} \mathbf{f}_{1}\right|^{2}}=\frac{\left|\mathbf{h}_{2}^{\mathrm{H}} \mathbf{f}_{\mathrm{c}}\right|^{2}}{\sigma_{2}^{2}+\left|\mathbf{h}_{2}^{\mathrm{H}} \mathbf{f}_{2}\right|^{2}}$ and $\left|\overline{\mathbf{h}}_{1}^{\mathrm{H}} \mathbf{f}_{\mathrm{c}}\right|^{2}=\left|\overline{\mathbf{h}}_{2}^{\mathrm{H}} \mathbf{f}_{\mathrm{c}}\right|^{2}$, and the solution of Problem (13) is $\mathbf{f}_{\mathrm{c}}=\sqrt{(1-t) p_{x}} \mathbf{v}_{\mathrm{c}}$ with precoding direction $\mathbf{v}_{\mathrm{c}}$,

$$
\mathbf{v}_{\mathrm{c}}=\frac{1}{\sqrt{2\left(1+\left|\mathbf{h}_{1}^{\mathrm{H}} \mathbf{h}_{2}\right|\right)}}\left(\overline{\mathbf{h}}_{1}+\overline{\mathbf{h}}_{2} e^{-j L \overline{\mathbf{h}}_{1}^{\mathrm{H}} \overline{\mathbf{h}}_{2}}\right) .
$$

The sum rate is expressed as $f\left(t, p_{x}\right)=\log _{2}(1+$ $\left.\beta_{1} p_{1}\right)+\log _{2}\left(1+\beta_{2} p_{2}\right)+R_{\mathrm{c}}$, where $R_{\mathrm{c}}=\min \left(\log _{2}(1+\right.$ $\left.\left.\frac{p_{\mathrm{c}}\left|\mathbf{h}_{1}^{\mathrm{H}} \mathbf{v}_{\mathrm{c}}\right|^{2}}{\sigma_{1}^{2}+p_{1}\left|\mathbf{h}_{1}^{\mathrm{H}} \mathbf{v}_{1}\right|^{2}}\right), \log _{2}\left(1+\frac{p_{\mathrm{c}}\left|\mathbf{h}_{2}^{\mathrm{H}} \mathbf{v}_{\mathrm{c}}\right|^{2}}{\sigma_{2}^{2}+p_{2}\left|\mathbf{h}_{2}^{\mathrm{H}} \mathbf{v}_{2}\right|^{2}}\right)\right)$. Owing to the equation $\log _{2}\left(1+\frac{p_{\mathrm{c}}\left|\mathbf{h}_{1}^{\mathrm{H}} \mathbf{v}_{\mathrm{c}}\right|^{2}}{\sigma_{1}^{2}+p_{1}\left|\mathbf{h}_{1}^{\mathrm{H}} \mathbf{v}_{1}\right|^{2}}\right)=\log _{2}\left(1+\frac{p_{\mathrm{c}}\left|\mathbf{h}_{2}^{\mathrm{H}} \mathbf{v}_{\mathrm{c}}\right|^{2}}{\sigma_{2}^{2}+p_{2}\left|\mathbf{h}_{2}^{\mathrm{H}} \mathbf{v}_{2}\right|^{2}}\right)$, we can choose $R_{\mathrm{c}}=\log _{2}\left(1+\frac{p_{\mathrm{c}}\left|\mathbf{h}_{2}^{\mathrm{H}} \mathbf{v}_{\mathrm{c}}\right|^{2}}{\sigma_{2}^{2}+p_{2}\left|\mathbf{h}_{2}^{\mathrm{H}} \mathbf{v}_{2}\right|^{2}}\right)$, and rewrite the sum rate as

$f\left(t, p_{x}\right)=\log _{2}\left(1+\beta_{1} p_{1}\right)+\log _{2}\left(1+\beta_{2} p_{2}+\beta_{\mathrm{c}}(1-t) p_{x}\right)$

where $\beta_{\mathrm{c}}=\frac{\left|\mathbf{h}_{2}^{\mathrm{H}} \mathbf{v}_{\mathrm{c}}\right|^{2}}{\sigma_{2}^{2}}$.

Substituting (11) into (15), we can write

$f\left(t, p_{x}\right)=\log _{2}\left(\beta_{1} \mu\left(t, p_{x}\right)\right)+\log _{2}\left(\beta_{2} \mu\left(t, p_{x}\right)+\beta_{\mathrm{c}}(1-t) p_{x}\right)$.

The optimal $t$ that maximizes $f\left(t, p_{x}\right)$ is obtained by setting $\frac{\partial f\left(t, p_{x}\right)}{\partial t}=0$, which results

$t= \begin{cases}\frac{1}{2 \beta_{\mathrm{c}}-\beta_{2}}\left(\frac{\left(\beta_{2}-\beta_{\mathrm{c}}\right)\left(\beta_{1}+\beta_{2}\right)}{\beta_{1} \beta_{2} p_{x}}+\beta_{\mathrm{c}}\right), & \text { if } \frac{1}{2} \leq\left|\overline{\mathbf{h}}_{1}^{\mathrm{H}} \overline{\mathbf{h}}_{2}\right| \leq 1 \\ 1, & \text { if } 0 \leq\left|\overline{\mathbf{h}}_{1}^{\mathrm{H}} \overline{\mathbf{h}}_{2}\right|<\frac{1}{2}\end{cases}$

which is due to $t-1=\frac{\beta_{2}-\beta_{\mathrm{c}}}{2 \beta_{\mathrm{c}}-\beta_{2}}\left(\frac{\beta_{1}+\beta_{2}}{\beta_{1} \beta_{2} p_{x}}+1\right), 2 \beta_{\mathrm{c}}-\beta_{2}>0$, and

$$
\left(\beta_{2}-\beta_{\mathrm{c}}\right) \begin{cases}\leq 0, & \text { if } \frac{1}{2} \leq\left|\overline{\mathbf{h}}_{1}^{\mathrm{H}} \overline{\mathbf{h}}_{2}\right| \leq 1 \\ >0, & \text { if } 0 \leq\left|\overline{\mathbf{h}}_{1}^{\mathrm{H}} \overline{\mathbf{h}}_{2}\right|<\frac{1}{2}\end{cases}
$$

The solution in 17) maximizing SE is the same with the solution in (13) in [37]. According to (17), when $0 \leq$ $\left|\overline{\mathbf{h}}_{1}^{\mathrm{H}} \overline{\mathbf{h}}_{2}\right|<\frac{1}{2}$, RSMA boils down to SDMA $(t=1)$.

Substituting (17) into 111, then the transmit power of each stream is

$p_{1}=\max \left(\frac{\beta_{\mathrm{c}}}{2\left(2 \beta_{\mathrm{c}}-\beta_{2}\right)}\left(\frac{2 \beta_{2}}{\beta_{1} \beta_{\mathrm{c}}}+\frac{1}{\beta_{2}}-\frac{3}{\beta_{1}}+p_{x}\right), 0\right)$,

$p_{2}=\max \left(\frac{\beta_{\mathrm{c}}}{2\left(2 \beta_{\mathrm{c}}-\beta_{2}\right)}\left(\frac{2}{\beta_{\mathrm{c}}}+\frac{1}{\beta_{1}}-\frac{3}{\beta_{2}}+p_{x}\right), 0\right)$.

Furthermore, substituting (18) into (15), the sum rate is expressed as

$$
\begin{aligned}
f\left(p_{x}\right)= & \log _{2}\left(1+\beta_{1} p_{1}\right) \\
& +\log _{2}\left(1+\beta_{2} p_{2}+\beta_{\mathrm{c}}\left(p_{x}-p_{1}-p_{2}\right)\right) \\
= & \log _{2}\left(\frac{\beta_{\mathrm{c}} \beta_{1}}{2\left(2 \beta_{\mathrm{c}}-\beta_{2}\right)}\left(p_{x}+\frac{1}{\beta_{1}}+\frac{1}{\beta_{2}}\right)\right) \\
& +\log _{2}\left(\frac{\beta_{\mathrm{c}}}{2}\left(p_{x}+\frac{1}{\beta_{1}}+\frac{1}{\beta_{2}}\right)\right) .
\end{aligned}
$$

With 19], the SE-EE tradeoff problem in 10] is reformulated as

$$
\begin{aligned}
& \max _{p_{x}} L\left(p_{x}\right)=w \frac{f\left(p_{x}\right)}{p_{x}+\chi f\left(p_{x}\right)+\mathrm{P}_{\mathrm{c}}}+(1-w) \frac{f\left(p_{x}\right)}{\mathrm{P}_{\mathrm{c}}} \\
& \text { s.t. } 0 \leq p_{x} \leq \mathrm{P}_{\max } .
\end{aligned}
$$

Problem 201 is a convex problem with the concave objective function $L\left(p_{x}\right)$ and the affine constraint. The first-order derivative of $L\left(p_{x}\right)$ is a monotone decreasing function of $p_{x}$, and is expressed as

$$
L^{\prime}\left(p_{x}\right)=\frac{f^{\prime}\left(p_{x}\right)}{\mathrm{P}_{\mathrm{c}}}\left(1-\left(\frac{z_{1}\left(p_{x}\right)}{z_{2}\left(p_{x}\right)}+1\right) w\right),
$$


where $f^{\prime}\left(p_{x}\right)=\frac{2}{\ln 2\left(p_{x}+\frac{1}{\beta_{1}}+\frac{1}{\beta_{2}}\right)}>0, z_{1}\left(p_{x}\right)=\mathrm{P}_{\mathrm{c}}\left(f\left(p_{x}\right)-\right.$ $\left.f^{\prime}\left(p_{x}\right) p_{x}-f^{\prime}\left(p_{x}\right) \mathrm{P}_{\mathrm{c}}\right)$, and $z_{2}\left(p_{x}\right)=f^{\prime}\left(p_{x}\right)\left(p_{x}+\chi f\left(p_{x}\right)+\right.$ $\left.\mathrm{P}_{\mathrm{C}}\right)^{2}>0$. When $w=1$, we denote $\widetilde{p}_{w=1}$ which meets $L^{\prime}\left(\widetilde{p}_{w=1}\right)=0$ and $z_{1}\left(\tilde{p}_{w=1}\right)=0$. Since $-z_{1}^{\prime}\left(p_{x}\right)=$ $\mathrm{P}_{\mathrm{c}} f^{\prime \prime}\left(p_{x}\right)\left(p_{x}+\mathrm{P}_{\mathrm{c}}\right)<0$ with $f^{\prime \prime}\left(p_{x}\right)=\frac{-2}{\left(\ln 2\left(p_{x}+\frac{1}{\beta_{1}}+\frac{1}{\beta_{2}}\right)\right)^{2}}<$ 0 , then $z_{1}\left(p_{x}\right)$ is a monotone decreasing function of $p_{x}$. The minimum value of $L^{\prime}\left(p_{x}\right)$ is obtained at $p_{x}=\mathrm{P}_{\max }$. If $\mathrm{P}_{\max } \leq \widetilde{p}_{w=1}$, we always have $L^{\prime}\left(\mathrm{P}_{\max }\right) \geq 0$, then the optimal $p_{x}$ is $\mathrm{P}_{\max }$. If $\mathrm{P}_{\max }>\widetilde{p}_{w=1}$, we have $L^{\prime}\left(\mathrm{P}_{\max }\right)>0$ when $0 \leq w<\frac{z_{2}\left(\mathrm{P}_{\max }\right)}{z_{1}\left(\mathrm{P}_{\max }\right)+z_{2}\left(\mathrm{P}_{\max }\right)}$, then the optimal $p_{x}$ is $\mathrm{P}_{\max }$. If $\mathrm{P}_{\max }>\widetilde{p}_{w=1}$, we have $L^{\prime}\left(\mathrm{P}_{\max }\right) \leq 0$ when $\frac{z_{2}\left(\mathrm{P}_{\max }\right)}{z_{1}\left(\mathrm{P}_{\max }\right)+z_{2}\left(\mathrm{P}_{\max }\right)} \leq w \leq 1$, then the optimal $p_{x}$ is the solution of $L^{\prime}\left(p_{x}\right)=0$. Denote $\widetilde{p}$ such that $L^{\prime}(\widetilde{p})=0$ when $\mathrm{P}_{\max }>\widetilde{p}_{w=1} \cdot \widetilde{p}_{w=1}$ and $\widetilde{p}$ can be obtained by "solve" function in MATLAB. Please note that $\widetilde{p}_{w=1}$ and $\widetilde{p}$ is globle optimal due to the concave function $L\left(p_{x}\right)$. Based on the above analysis, the optimal solution of Problem (20) is given by

$\mathrm{P}_{\text {opt }}= \begin{cases}\mathrm{P}_{\max }, & \text { if } \mathrm{P}_{\max } \leq \widetilde{p}_{w=1} \\ \mathrm{P}_{\max }, & \text { if } \mathrm{P}_{\max }>\widetilde{p}_{w=1} \text { and } 0 \leq w<\frac{1}{\frac{z_{1}\left(\mathrm{P}_{\max }\right)}{z_{2}\left(\mathrm{P}_{\max }\right)}+1} \\ \widetilde{p}, & \text { if } \mathrm{P}_{\max }>\widetilde{p}_{w=1} \text { and } \frac{1}{\frac{z_{1}\left(\mathrm{P}_{\max }\right)}{z_{2}\left(\mathrm{P}_{\max }\right)}+1} \leq w \leq 1 .\end{cases}$

The corresponding optimal sum rate and the energy efficiency are directly given by $\mathrm{SE}=f\left(\mathrm{P}_{\mathrm{opt}}\right)$ and $\mathrm{EE}=$ $\frac{f\left(\mathrm{P}_{\mathrm{opt}}\right)}{+\chi f\left(\mathrm{P}_{\mathrm{opt}}\right)+\mathrm{P}_{\mathrm{c}}}$, respectively.

Remark 2: Following from (21), we would like to highlight that the optimal transmit power $\mathrm{P}_{\text {opt }}$ of the SE-EE tradeoff is controlled by two factors, namely, the maximum transmit power limit $\mathrm{P}_{\max }$ at the $\mathrm{BS}$ and the weight $w$ allocated to EE and SE. When $\mathrm{P}_{\max } \leq \widetilde{p}_{w=1}, \mathrm{SE}$ and EE are both monotonic increasing function of the transmit power for all weights in $0 \leq w \leq 1$. In contrast, when $\mathrm{P}_{\max }>\widetilde{p}_{w=1}$, $\mathrm{EE}$ and SE are conflicting with each other as the transmit power increases. Therefore, such result helps us further show the necessity of investigating the SE-EE tradeoff problem when $\mathrm{P}_{\max }>\widetilde{p}_{w=1}$, and the optimal $\mathrm{P}_{\text {opt }}$ that achieves the optimal SE-EE tradeoff is adjusted by $w$.

\section{B. SCA-based Algorithm for $K$-user system}

Problem 10 for the $K$-user system is difficult to solve for the reason that the objective function has non-convex numerator and denominator, and the non-convex property mainly comes from the rate expressions. A popular method to solve general fractional programming problem is Dinkelbach's method [24]. It is capable of converting fractional programming to linear programming by introducing parameters to denote those fractions. These parametric linear programming problems are then addressed by applying SCA [39] and WMMSE [19]. The Dinkelbach's framework is in fact a two-layer iterative procedure, which requires huge computational complexity. More importantly, the convergence of this framework cannot be guaranteed since each parametric linear programming problem may only achieve local optimum [26].
In order to address the above shortcomings, we propose a one-layer iterative algorithm under the SCA framework [25] directly. Firstly, we introduce a new variable $\eta$ denoting the $\mathrm{EE}$ and reformulate Problem (10) as

$$
\begin{aligned}
& \max _{\mathbf{F} \in \mathcal{S}, \eta} w \eta+(1-w) \frac{f(\mathbf{F})}{\mathrm{P}_{\mathrm{c}}} \\
& \text { s.t. } \quad \eta \leq \frac{f(\mathbf{F})}{g(\mathbf{F})} .
\end{aligned}
$$

To tractably recast non-convex fractional constraint 22b, we replace it with the following three constraints

$$
\begin{aligned}
\eta & \leq \frac{x^{2}}{y}, \\
x^{2} & \leq f(\mathbf{F}), \\
g(\mathbf{F}) & \leq y,
\end{aligned}
$$

where variable $x$ represents the square roof of the total sum rate, and variable $y$ represents the total power. Then, we introduce variables $\mathbf{r}=\left[r_{\mathrm{c}}, r_{1}, \ldots, r_{K}\right]^{\mathrm{T}}$ to denote rates $\left\{R_{i}(\mathbf{F})\right\}_{\forall i \in \mathcal{K}_{\mathrm{c}}}$ and rewrite Problem (22) equivalently as

$$
\begin{array}{ll}
\max _{\mathbf{f} \in \mathcal{S}, \eta, \mathbf{r}, x, y} & w \eta+\frac{(1-w)}{\mathrm{P}_{\mathrm{c}}} \sum_{i \in \mathcal{K}_{\mathrm{c}}} r_{i} \\
\text { s.t. } \quad & \quad \underline{23 \mathrm{a}} \\
& x^{2} \leq \sum_{i \in \mathcal{K}_{\mathrm{c}}} r_{i} \\
& \|\mathbf{F}\|_{F}^{2}+\mathrm{P}_{\mathrm{c}}+\chi \sum_{i \in \mathcal{K}_{\mathrm{c}}} r_{i} \leq y \\
& r_{k} \leq R_{k}(\mathbf{F}), \forall k \in \mathcal{K} \\
& r_{\mathrm{c}} \leq R_{\mathrm{c}, k}(\mathbf{F}), \forall k \in \mathcal{K}
\end{array}
$$

The non-convexity of Problem 24a is due to the constraints 23a, 24e and 24f, which motivates us to use SCA framework to approximate the non-convex constraints. Specifically the right hand side of (23a) is a quadraticover-linear function, which is jointly convex in $(x, y)$. We approximate it by its first-order lower approximation at fixed point $\left(x^{(n)}, y^{(n)}\right)$ as [40]

$$
\frac{x^{2}}{y} \geq \frac{2 x^{(n)}}{y^{(n)}} x-\left(\frac{x^{(n)}}{y^{(n)}}\right)^{2} y \triangleq \phi^{(n)}(x, y) .
$$

The remaining challenge is to tackle the non-convexity of constraints 24e and 24f). In most literature, the relation between rate and WMMSE is used to transform the nonconvex rate-based function into its convex WMMSE counterpart by introducing auxiliary variables, i.e., weights and equalizers. The method known as WMMSE is widely used in the literature [12], [19], [25] and shows good performance. However, WMMSE method is an iterative optimization method, in which the weights, equalizers and precoders are updated in an iterative manner.

In the following, we investigate the intrinsic convexity of the rate expressions $R_{k}(\mathbf{F})$ and $R_{\mathrm{c}, k}(\mathbf{F})$, and then propose two lower bounds to approximate the non-convex rate expressions by using the SCA method. 
1) Lower-bound ( $L B) I$ : The first lower bound of rate is summarized in the following Lemma 1

Lemma 1 Let $\mathbf{F}^{(n)}$ denote the optimal solution obtained in the $(n-1)$-th iteration. The concave lower bound function of $R_{k}(\mathbf{F})$ in the $n$-th iteration is given by

$$
R_{k}^{(n)}(\mathbf{F}) \triangleq \text { const }_{k}+2 \operatorname{Re}\left\{a_{k} \mathbf{b}_{k}^{\mathrm{H}} \mathbf{f}_{k}\right\}-a_{k} \sum_{i \in \mathcal{K}} \mathbf{f}_{i}^{\mathrm{H}} \mathbf{b}_{k} \mathbf{b}_{k}^{\mathrm{H}} \mathbf{f}_{i},
$$

at point $\mathbf{F}^{(n)}$, where

$$
\begin{gathered}
a_{k}=1+\left(\sigma_{k}^{2}+\sum_{i \in \mathcal{K} \backslash\{k\}} \mathbf{h}_{k}^{\mathrm{H}} \mathbf{f}_{i}^{(n)} \mathbf{f}_{i}^{(n), \mathrm{H}} \mathbf{h}_{k}\right)^{-1} \mathbf{f}_{k}^{(n), \mathrm{H}} \mathbf{h}_{k} \mathbf{h}_{k}^{\mathrm{H}} \mathbf{f}_{k}^{(n)}, \\
\mathbf{b}_{k}=\left(\sigma_{k}^{2}+\sum_{i \in \mathcal{K}} \mathbf{h}_{k}^{\mathrm{H}} \mathbf{f}_{i}^{(n)} \mathbf{f}_{i}^{(n), \mathrm{H}} \mathbf{h}_{k}\right)^{-1} \mathbf{h}_{k}^{\mathrm{H}} \mathbf{f}_{k}^{(n)} \mathbf{h}_{k}, \\
\operatorname{const}_{k}=R_{k}\left(\mathbf{F}^{(n)}\right)-2 \operatorname{Re}\left\{a_{k} \mathbf{b}_{k}^{\mathrm{H}} \mathbf{f}_{k}^{(n)}\right\} \\
+a_{k} \sum_{i \in \mathcal{K}} \mathbf{f}_{i}^{(n), \mathrm{H}} \mathbf{b}_{k} \mathbf{b}_{k}^{\mathrm{H}} \mathbf{f}_{i}^{(n)} .
\end{gathered}
$$

Meanwhile, the concave lower bound function of $R_{\mathrm{c}, k}(\mathbf{F})$ is given by

$$
\begin{aligned}
& R_{\mathrm{c}, k}^{(n)}(\mathbf{F}) \triangleq \\
& \operatorname{const}_{\mathrm{c}, k}+2 \operatorname{Re}\left\{a_{\mathrm{c}, k} \mathbf{b}_{\mathrm{c}, k}^{\mathrm{H}} \mathbf{f}_{\mathrm{c}}\right\}-a_{\mathrm{c}, k} \sum_{i \in \mathcal{K}_{\mathrm{c}}} \mathbf{f}_{i}^{\mathrm{H}} \mathbf{b}_{\mathrm{c}, k} \mathbf{b}_{\mathrm{c}, k}^{\mathrm{H}} \mathbf{f}_{i},
\end{aligned}
$$

at point $\mathbf{f}^{n}$, where

$$
\begin{gathered}
a_{\mathrm{c}, k}=1+\left(\sigma_{k}^{2}+\sum_{i \in \mathcal{K}} \mathbf{h}_{k}^{\mathrm{H}} \mathbf{f}_{i}^{(n)} \mathbf{f}_{i}^{(n), \mathrm{H}} \mathbf{h}_{k}\right)^{-1} \mathbf{f}_{\mathrm{c}}^{(n), \mathrm{H}} \mathbf{h}_{k} \mathbf{h}_{k}^{\mathrm{H}} \mathbf{f}_{\mathrm{c}}^{(n)}, \\
\mathbf{b}_{\mathrm{c}, k}=\left(\sigma_{k}^{2}+\sum_{i \in \mathcal{K}_{\mathrm{c}}} \mathbf{h}_{k}^{\mathrm{H}} \mathbf{f}_{i}^{(n)} \mathbf{f}_{i}^{(n), \mathrm{H}} \mathbf{h}_{k}\right)^{-1} \mathbf{h}_{k}^{\mathrm{H}} \mathbf{f}_{\mathrm{c}}^{(n)} \mathbf{h}_{k}, \\
\text { const }_{\mathrm{c}, k}=R_{\mathrm{c}, k}\left(\mathbf{F}^{(n)}\right)-2 \operatorname{Re}\left\{a_{\mathrm{c}, k} \mathbf{b}_{\mathrm{c}, k}^{\mathrm{H}} \mathbf{f}_{\mathrm{c}}^{(n)}\right\} \\
+a_{\mathrm{c}, k} \sum_{i \in \mathcal{K}_{\mathrm{c}}} \mathbf{f}_{i}^{(n), \mathrm{H}} \mathbf{b}_{\mathrm{c}, k} \mathbf{b}_{\mathrm{c}, k}^{\mathrm{H}} \mathbf{f}_{i}^{(n)} .
\end{gathered}
$$

Proof: Please refer to Appendix $\mathrm{A}$

With the concave lower bound approximations (25), 28), and (26), Problem (24) is reformulated equivalently as the following convex problem:

$$
\begin{array}{rl}
\max _{\mathbf{F} \in \mathcal{S}, \eta, \mathbf{r}, x, y} & w \eta+\frac{(1-w)}{\mathrm{P}_{\mathrm{c}}} \sum_{i \in \mathcal{K}_{\mathrm{c}}} r_{i} \\
\text { s.t. } \quad & \eta \leq \phi^{(n)}(x, y) \\
& r_{k} \leq R_{k}^{(n)}(\mathbf{F}), \forall k \in \mathcal{K} \\
& r_{\mathrm{c}} \leq R_{\mathrm{c}, k}^{(n)}(\mathbf{F}), \forall k \in \mathcal{K} \\
& \text { 24c), 24d. } .
\end{array}
$$

2) Lower-bound (LB) II: We note that the lower bound approximation proposed in Lemma 1 is the first-order approximation of $\log$ function directly. Recall that each rate function is a composition function with an inner fractional SINR function embraced by an outer log function. Motivated by the convexity of the outer log function, another method is to approximate only the inner SINR function with its concave lower bound and keep the outer $\log$ function. Specifically, we introduce new variables $\gamma=\left[\gamma_{1}, \ldots, \gamma_{K}\right]^{\mathrm{T}}$ to denote the SINRs of the private streams at all users and variables $\gamma_{\mathrm{c}}=\left[\gamma_{\mathrm{c}, 1}, \ldots, \gamma_{\mathrm{c}, K}\right]^{\mathrm{T}}$ to denote the SINRs of the common streams at all users. Constraints 24e and (24f can be recast equivalently as

$$
\begin{aligned}
& r_{k} \leq \log _{2}\left(1+\gamma_{k}\right), \forall k \in \mathcal{K} \\
& r_{\mathrm{c}} \leq \log _{2}\left(1+\gamma_{\mathrm{c}, k}\right), \forall k \in \mathcal{K} \\
& \gamma_{k} \leq \frac{\left|\mathbf{h}_{k}^{\mathrm{H}} \mathbf{f}_{k}\right|^{2}}{I_{-k}}, \forall k \in \mathcal{K} \\
& \gamma_{c, k} \leq \frac{\left|\mathbf{h}_{k}^{\mathrm{H}} \mathbf{f}_{\mathrm{c}}\right|^{2}}{I_{k}}, \forall k \in \mathcal{K}
\end{aligned}
$$

where $I_{-k}=\sigma_{k}^{2}+\sum_{i \in \mathcal{K} \backslash\{k\}} \mathbf{h}_{k}^{\mathrm{H}} \mathbf{f}_{i} \mathbf{f}_{i}^{\mathrm{H}} \mathbf{h}_{k}$ and $I_{k}=I_{-k}+$ $\mathbf{h}_{k}^{\mathrm{H}} \mathbf{f}_{k} \mathbf{f}_{k}^{\mathrm{H}} \mathbf{h}_{k}$. The right hand side of (31c) and (31d) are all in the form of $\frac{x^{2}}{y}$. Thus, according to (25), we obtain (32) by applying substitutions $x=\mathbf{h}_{k}^{\mathrm{H}} \mathbf{f}_{k}$ and $y=I_{-k}$, and (33) by applying substitutions $x=\mathbf{h}_{k}^{\mathrm{H}} \mathbf{f}_{\mathrm{c}}$ and $y=I_{k}$.

$$
\frac{\left|\mathbf{h}_{k}^{\mathrm{H}} \mathbf{f}_{k}\right|^{2}}{I-k} \geq \frac{2 \operatorname{Re}\left\{\mathbf{f}_{k}^{n, \mathrm{H}} \mathbf{h}_{k} \mathbf{h}_{k}^{\mathrm{H}} \mathbf{f}_{k}\right\}}{I_{-k}^{n}}-\left|\frac{\mathbf{h}_{k}^{\mathrm{H}} \mathbf{f}_{k}^{n}}{I_{-k}^{n}}\right|^{2} I_{-k} \triangleq \Gamma_{k}^{(n)}(\mathbf{F}),
$$

$$
\frac{\left|\mathbf{h}_{k}^{\mathrm{H}} \mathbf{f}_{\mathrm{c}}\right|^{2}}{I_{k}} \geq \frac{2 \operatorname{Re}\left\{\mathbf{f}_{\mathrm{c}}^{n, \mathrm{H}} \mathbf{h}_{k} \mathbf{h}_{k}^{\mathrm{H}} \mathbf{f}_{\mathrm{c}}\right\}}{I_{k}^{n}}-\left|\frac{\mathbf{h}_{k}^{\mathrm{H}} \mathbf{f}_{k}^{n}}{I_{k}^{n}}\right|^{2} I_{k} \triangleq \Gamma_{\mathrm{c}, k}^{(n)}(\mathbf{F}) .
$$

With 31a, 31b, and the concave lower bound approximations (32) and (33), Problem 24a) is reformulated equivalently as the following convex problem:

$$
\begin{array}{rl}
\max _{\mathbf{F} \in \mathcal{S}, \eta, \mathbf{r}, x, y, \boldsymbol{\gamma}, \boldsymbol{\gamma}_{\mathrm{c}}} & w \eta+\frac{(1-w)}{\mathrm{P}_{\mathrm{c}}} \sum_{i \in \mathcal{K}_{\mathrm{c}}} r_{i} \\
\text { s.t. } \quad & \gamma_{k} \leq \Gamma_{k}^{(n)}(\mathbf{F}), \forall k \in \mathcal{K} \\
& \gamma_{\mathrm{c}, k} \leq \Gamma_{\mathrm{c}, k}^{(n)}(\mathbf{F}), \forall k \in \mathcal{K} \\
& (24 c),(24 d),(30 b), \text { (31a), (31b). }
\end{array}
$$

LB II approximating only the inner SINR function is tighter than LB I which is the lower bound of the outer rate function. Consequently, LB II requires less number of iterations for convergence. In the following, we derive algorithms based on the specified LB I or LB II, and the relation between LB I and LB II can be reflected clearly in the simulation. The optimal SE-EE tradeoff problem can be achieved by designing $\mathbf{f}$ under the SCA framework which is summarized in Algorithm 1 . The convex Problem (30) and (34) are solved by the standard interior-point algorithm [40]. The initial points are generated as follows. $\mathbf{F}^{(0)}$ is created 
to meet the power constraint $\mathbf{F} \in \mathcal{S}$, and then $x^{(0)}$ and $y^{(0)}$ are obtained by setting constraints (23b) and 23c to be equality, respectively.

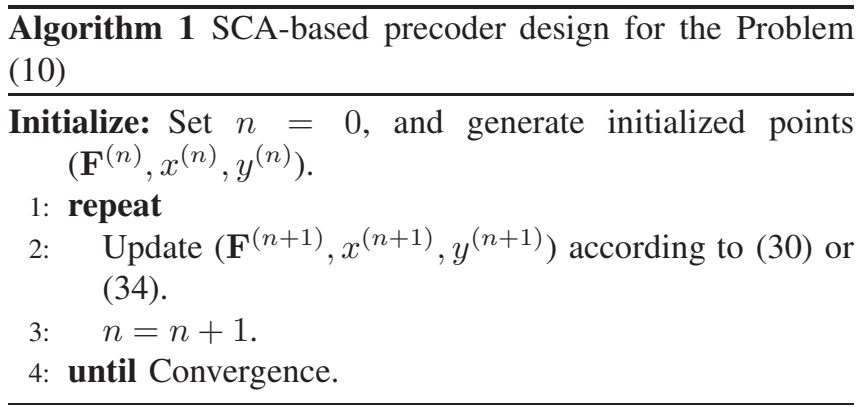

3) Convergence and Complexity Analysis: In this subsection, we discuss the convergence and per-iteration complexity of Algorithm 1 To start with, the optimal solution of Problem (30) or 34) obtained at the $n$-th iteration is also feasible at the next iteration [41]. Therefore, the sequence of the objective values generated by Algorithm 1 is nondecreasing and the sequence is bounded above due to the power constraints $\mathbf{F} \in \mathcal{S}$. Hence, the convergence of Algorithm 1 is guaranteed. Moreover, the following proposition shows the solution property of Algorithm 1 .

Proposition 1 Denote by $\mathbf{F}^{o}$ the converged solution of Algorithm 1 then $\mathbf{F}^{o}$ converges to a KKT point of Problem (10).

Proof: Please refer to Appendix B

Next, we estimate the worst-case per-iteration complexity of Algorithm 1 with the second-order cone programming (SOCP) (30) and general convex program (GCP) (34). Specifically, the computational complexity of solving SOCP is $\mathcal{O}\left(N_{\text {socp }} M_{\text {socp }}^{3.5}+N_{\text {socp }}^{3} M_{\text {socp }}^{2.5}\right)$, where $N_{\text {socp }}$ and $M_{\text {socp }}$ are the dimension of second order cone and the number of second order cone constraints, respectively. Therefore, the per-iteration computational complexity of solving SOCP (30) is $\mathcal{O}\left(N_{t}(K+1)^{4.5}+N_{t}^{3}(K+1)^{5.5}\right)$. Meanwhile, the per-iteration computational complexity of solving the GCP (34) is $\mathcal{O}\left(N_{t}^{4}(K+1)^{4}\right)$.

\section{Weighted-PoWER APPROACH}

In the low transmit power constraint $P_{\max }$, both EE and SE increase with $P_{\max }$, and the optimal transmit power equals $P_{\max }$. Therefore, there is no conflict of interest between these two objective functions. However, when $P_{\max }$ is large, the maximum $\mathrm{SE}$ is achieved when the optimal transmit power equals $P_{\max }$, which reduces the EE. In order to maximize EE, only part of the available power is used, which reduces the SE eventually. That means there is a tradeoff between EE and SE moderate and high SNR regimes. Since both EE and SE are affected by transmit power, a weighted-power EE metric is proposed to investigate this tradeoff.
According to [21], maximizing EE and SE is also equivalent to minimizing their inverse. Therefore Problem 90 is equivalent to

$$
\min _{\mathbf{F} \in \mathcal{S}}\left[\frac{g_{\mathrm{RS}}(\mathbf{F})}{f_{\mathrm{RS}}(\mathbf{F})}, \frac{1}{f_{\mathrm{RS}}(\mathbf{F})}\right] .
$$

Problem 35 is also solved via its corresponding singleobjective problem as follows

$$
\min _{\mathbf{F} \in \mathcal{S}} w \frac{g_{\mathrm{RS}}(\mathbf{F})}{f_{\mathrm{RS}}(\mathbf{F})}+(1-w) \frac{\mathrm{P}_{\mathrm{c}}}{f_{\mathrm{RS}}(\mathbf{F})},
$$

where $w \in[0,1]$. With the same denominator, Problem 36 is equivalent to

$$
\max _{\mathbf{F} \in \mathcal{S}} \frac{f_{\mathrm{RS}}(\mathbf{F})}{w\left(\|\mathbf{F}\|_{F}^{2}+\chi f_{\mathrm{RS}}(\mathbf{F})\right)+\mathrm{P}_{\mathrm{c}}} .
$$

From a mathematical point of view, the metric in Problem (37) only has an additional constant $w$ in the denominator compared with Problem (8). Hence, we name the objective of Problem 37 weighted-power EE metric. Physically speaking, by changing $w$ from 0 to 1 , we could investigate the SE-EE tradeoff. When $w=0$, Problem (37) focuses only on maximizing the SE without considering how much energy is consumed. When $w$ increases, it means there is a penalty for increasing the SNR. This process may reduce the power consumption and thereby reduce the SE. When $w=1$, Problem 37 focuses only on maximizing the EE.

When $K=2$, a closed-form solution of Problem (37) can be obtained by setting $L\left(p_{x}\right)$ in Problem 201 as

$$
L_{2}\left(p_{x}\right)=\frac{f\left(p_{x}\right)}{w\left(p_{x}+\chi f\left(p_{x}\right)\right)+\mathrm{P}_{\mathrm{c}}} .
$$

(38) is concave and its first-order derivative is a monotone decreasing function of $p_{x}$. We have

$$
L_{[2]}^{\prime}\left(p_{x}\right)=\frac{z_{3}\left(p_{x}\right) w+f^{\prime}\left(p_{x}\right) \mathrm{P}_{\mathrm{c}}}{\left(w\left(p_{x}+\chi f\left(p_{x}\right)\right)+\mathrm{P}_{\mathrm{c}}\right)^{2}},
$$

where $z_{3}\left(p_{x}\right)=f^{\prime}\left(p_{x}\right) p_{x}-f\left(p_{x}\right)$ and $z_{4}\left(p_{x}\right)=f^{\prime}\left(p_{x}\right) \mathrm{P}_{\mathrm{c}}$.

Denote $\widetilde{p}_{w=1}^{[2]}$ such that $L_{[2]}^{\prime}\left(\widetilde{p}_{w=1}^{[2]}\right)=0$ and $z_{3}\left(\widetilde{p}_{w=1}^{[2]}\right)+$ $z_{4}\left(\widetilde{p}_{w=1}^{[2]}\right)=0$, when $w=1$. Following the same analysis of obtaining (21), the optimal solution of Problem 201 is given by

$\mathrm{P}_{\mathrm{opt}}^{[2]}= \begin{cases}\mathrm{P}_{\max }, & \text { if } \mathrm{P}_{\max } \leq \widetilde{p}_{w=1}^{[2]} \\ \mathrm{P}_{\max }, & \text { if } \mathrm{P}_{\max }>\widetilde{p}_{w=1}^{[2]} \text { and } 0 \leq w<\frac{-z_{4}\left(\mathrm{P}_{\max }\right)}{z_{3}\left(\mathrm{P}_{\max }\right)} \\ \widetilde{p}^{[2]}, & \text { if } \mathrm{P}_{\max }>\widetilde{p}_{w=1}^{2]} \text { and } \frac{-z_{4}\left(\mathrm{P}_{\max }\right)}{z_{3}\left(\mathrm{P}_{\max }\right)} \leq w \leq 1,\end{cases}$

where $\widetilde{p}^{[2]}$ is the solution of $L_{[2]}^{\prime}\left(\widetilde{p}^{[2]}\right)=0$ when if $\mathrm{P}_{\max }>$ $\tilde{p}_{w=1}^{[2]}$ and $\frac{-z_{4}\left(\mathrm{P}_{\max }\right)}{z_{3}\left(\mathrm{P}_{\max }\right)} \leq w \leq 1 . \tilde{p}_{w=1}^{[2]}$ and $\tilde{p}^{[2]}$ can be obtained by "solve" function in MATLAB.

For $K$-user system, following the same method of obtaining Problem (30) and 34, Problem 37) can also be equivalently approximated by a SCA problem. Specifically, let a new variable $\hat{\eta}$ represent the weighted-power EE 
objective value in Problem (37) and take the place of $\eta$ in the constraint $30 \mathrm{~b}$, i.e.,

$$
\hat{\eta} \leq \phi^{(n)}(x, y)
$$

Then constraint (24d) is also replaced by

$$
w\left(\|\mathbf{F}\|_{F}^{2}+\chi \sum_{i \in \mathcal{K}_{\mathrm{c}}} r_{i}\right)+\mathrm{P}_{\mathrm{c}} \leq y .
$$

In summary, Problem 37) can be approximated by LB I as an SOCP given by

$$
\max _{\mathbf{f} \in \mathcal{S}, \eta, \mathbf{r}, x, y} \hat{\eta}
$$

s.t. (24c), (30c), (30d), (41), (42),

or by using LB II as a GCP given by

$$
\max _{\mathbf{f} \in \mathcal{S}, \eta, \mathbf{r}, x, y, \gamma, \boldsymbol{\gamma}_{c}} \hat{\eta}
$$

s.t. (24c), (31a), (31b), (34b), (34c), (41), (42).

Remark 3: Tow different metrics are proposed to investigate the SE-EE tradeoff. The weighed-sum approach is very intuitive, because it directly studies the MOO problem (9) with EE metric and SE metric, and then solves the MOO problem through its corresponding SOO problem. The weighted-power approach is an indirect way. By controlling the proportion of power consumption in the denominator of the objective function in (37), we indirectly control the proportions of EE metric and SE metric in the original MOO problem (9).

Remark 4: SE Problem (6) and EE Problem (8) are special cases of SE-EE tradeoff Problem (10) and 37). In particular, when $w=0$, Problem (10) and (37) reduce to Problem (6). When $w=1$, Problem (10) and (37) boil down to Problem (8). Therefore, the proposed Algorithm 1 can be leveraged to solve individual SE and EE problems.

\section{Numerical Results ANd Discussions}

In this section, extensive numerical results are provided to evaluate the effectiveness of our proposed algorithm and the SE-EE tradeoff performance of RSMA. Without loss of generality, the BS is equipped with $N_{t}=4$ transmit antennas, the noise power is $\sigma_{k}^{2}=-20 \mathrm{dBm}$, the static circuit power consumption is $\mathrm{P}_{\mathrm{c}}=5 \mathrm{dBW}, \chi=0.1$ $\mathrm{W} /(\mathrm{bit} / \mathrm{s} / \mathrm{Hz})$, and the iterative procedure of all the algorithms considered in this section is terminated when the objective values between two subsequent iterations is less than $10^{-6}$. The signal-to-noise ratio (SNR) in the figures is defined as $10 \log _{10}\left(\mathrm{P}_{\max } / \sigma_{k}^{2}\right)$. Some specific simulation parameters are given according to different figures. According to [10], we consider the simplified geometric channel model as

$$
\mathbf{h}_{k}=\nu_{k}\left[1, e^{j \frac{2 \pi}{\lambda_{c}} d \cos \theta_{k}}, \ldots, e^{j \frac{2 \pi}{\lambda_{c}}\left(N_{t}-1\right) d \cos \theta_{k}}\right]^{\mathrm{T}},
$$

where $\nu_{k}$ is the gain of channel and characterizes channel disparity parameter, $\theta_{k}$ is the angle-of-departure (AoD) from BS to user $k$ and characterizes the correlation between channels, and the scalar $d$ is the interval of antennas and

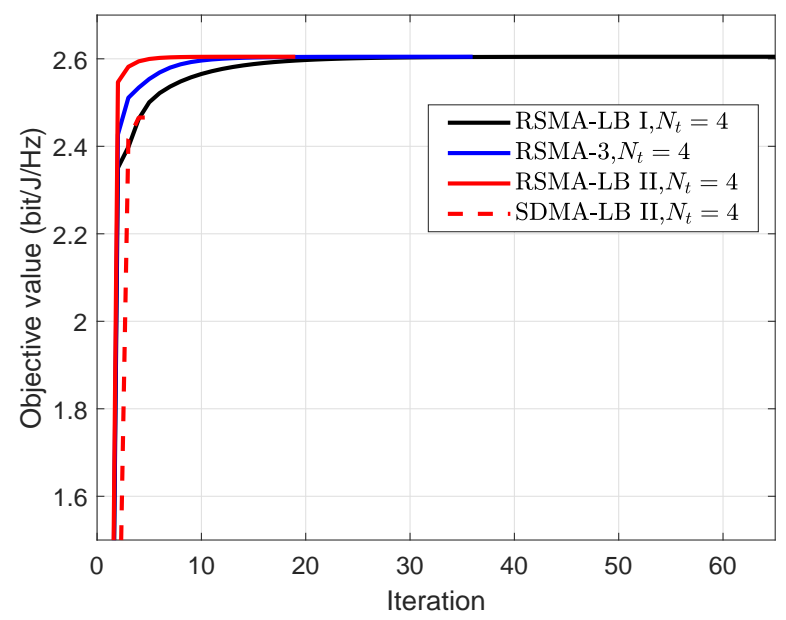

Fig. 2: Objective value versus number of iteration for RSMA/SDMA precoder designed by different SCA algorithms with $\mathrm{SNR}=20 \mathrm{~dB}, N_{t}=4, K=3$ and $w=0.5$.

$\lambda_{c}$ is the carrier wavelength. All algorithms are performed on a PC with a $1.99 \mathrm{GHz}$ i7-8550U CPU and 16 GB RAM, and all convex problems are solved by the advanced CVX tool, i.e., MOSEK solver [42].

\section{A. Convergence Analysis}

We note the fact that the computational complexity of Problem (30) and Problem (43) are the same, while that of Problem (34) and Problem (44) are also the same, hence this subsection only investigates the convergence performance of Problem (30) and Problem (34) proposed under weightedsum approach.

Denote the proposed low-complexity algorithm for twouser system as "RSMA-LC". Then, denote the proposed Algorithm 11 running with (30) and (34) as "RSMA-LB I" and "RSMA-LB II", respectively. As a comparison, an SOCP approximation of Problem (34) has been considered by replacing the exponential cone (31a and 31b with their convex approximations (see (43) in [23]) and we denote this SOCP approximation method as "RSMA-3". In addition, the Dinkelbach's algorithm proposed in [19] is also considered as our benchmark algorithm, which is denoted as "RSMA-D-MMSE". Basically, the idea of this benchmark algorithm is to use the WMMSE method to solve the parametric subproblems obtained from applying the Dinkelbach's algorithm to Problem (10). For completeness of the analysis, the SDMA is also considered, whose design problem can be solved by Dinkelbach's algorithm with semi-closed form solution [25] in each iteration or by (34) with minor modifications. Those methods are represented by "SDMA-D-bisearch" and "SDMA-LB II", respectively. The algorithms are summarized and compared in Table I.

Fig. 2 compares the numbers of iterations for those SCA algorithms with one layer iteration to converge when there are 3 users with $\left[\theta_{1}, \theta_{2}, \theta_{3}\right]=[0, \pi / 9,2 \pi / 9]$ and 
TABLE I: Comparison algorithm summary

\begin{tabular}{|c|c|c|c|}
\hline & System model & $\begin{array}{c}\text { Algorithm framework used to address the } \\
\text { fractional programming }\end{array}$ & Method used in the inner iteration \\
\hline \hline RSMA-LC & RSMA & Low-complexity & SOCP in [30] \\
\hline RSMA-LB I & RSMA & SCA & GCP in [34] \\
\hline RSMA-LB II & RSMA & SCA & SOCP approximation [23] of GCP in [34] \\
\hline RSMA-3 & RSMA & SCA & WMMSE [19] \\
\hline RSMA-D-MMSE & RSMA & Dinkelbach's & Semi-closed form solution with bisearch [25] \\
\hline SDMA-D-bisearch & SDMA & Dinkelbach's & GCP in [34] with $\left\|\mathbf{f}_{\mathrm{C}}\right\|^{2}=0$ \\
\hline SDMA-LB II & SDMA & SCA & GCP [4] with $\left\|\mathbf{f}_{\mathrm{C}}\right\| \|^{2}=0$ \\
\hline NOMA & NOMA & SCA & \\
\hline
\end{tabular}

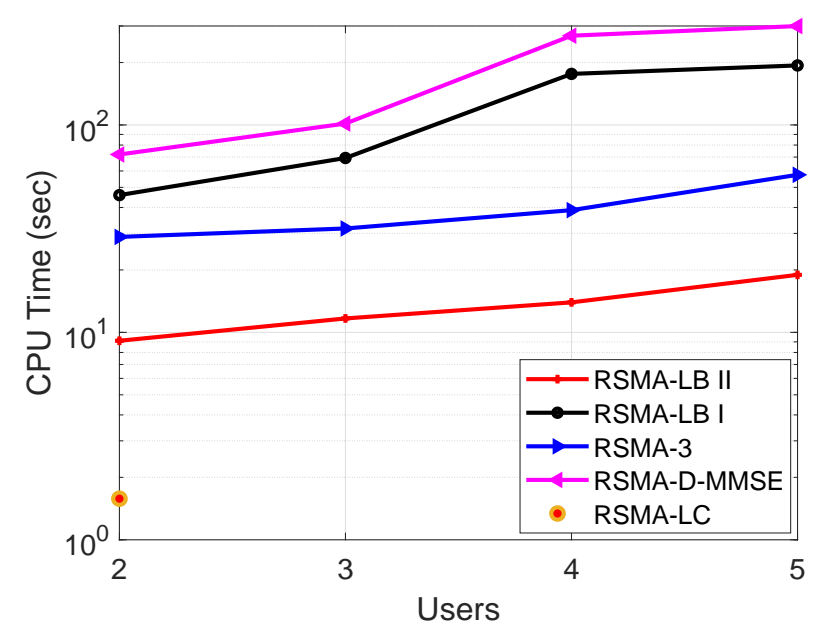

Fig. 3: CPU time versus $K$ by applying different algorithms with $\mathrm{SNR}=20 \mathrm{~dB}, N_{t}=4$ and $w=0.5$.

$\nu_{1}=\nu_{2}=\nu_{3}=1$. The SNR is $20 \mathrm{~dB}$ and the objective value in Y-axis is the optimal objective value of Problem (10). It is observed that the RSMA-LB II method takes the least number of iterations to converge since the LB II approximation is a tighter approximation of the original Problem (10) than LB I. But all optimization algorithms we proposed for RSMA converge to the same boundary point, which is higher than that of the SDMA.

Fig. 3 then compares the overall CPU time of different algorithms when there are 5 users under random channel realizations with the entries following i.i.d. CSCG distribution. We know that the CPU time of the proposed low-complexity algorithm is the lowest when $K=2$ due to the closed-form solution is obtained without iteration. When $K>2$, the proposed RSMA-LB II has the lowest complexity among all algorithms used for RSMA optimization.

\section{B. Energy Efficiency and Spectral Efficiency Performance of the RSMA}

The SE and EE benefits of RSMA have been verified in [4]. Specifically, [4] separately investigates the SE maximization problem which is solved by the WMMSE method and EE maximization problem which is solved by the SCA method. However, how the system EE of RSMA changes with the SNR, and how RSMA can control its SE and power

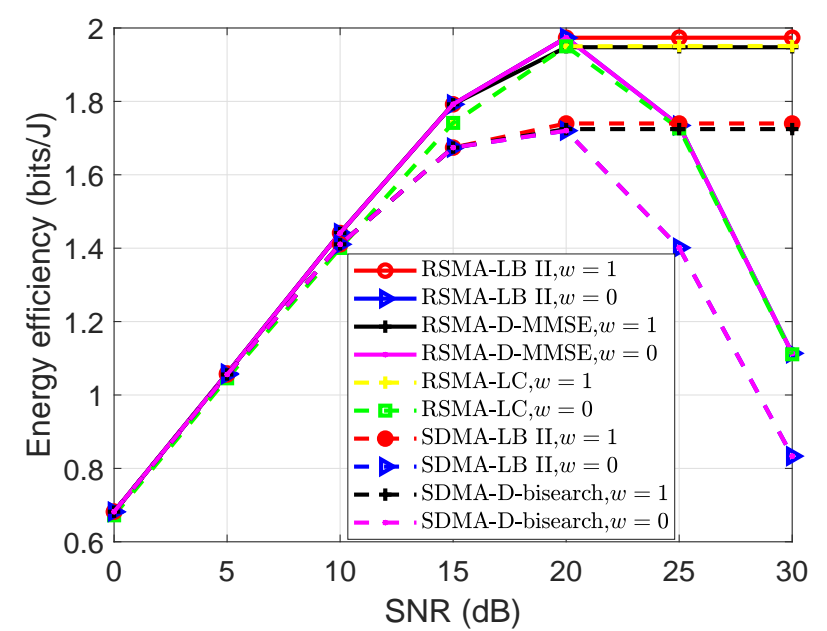

Fig. 4: Energy efficiency versus SNR for RSMA/SDMA precoder designed with $N_{t}=4$ and $K=2$.

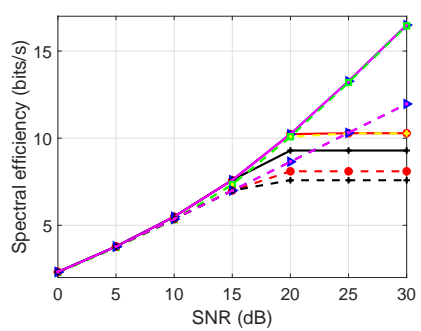

(a) Spectral efficiency versus SNR

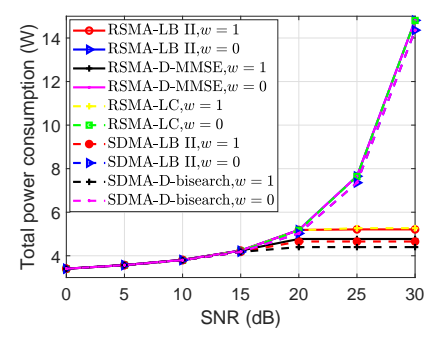

(b) Power consumption versus SNR
Fig. 5: Spectral efficiency and power consumption versus SNR for RSMA/SDMA precoder designed with $N_{t}=4$ and $K=2$.

consumption to achieve high EE, have never been studied. In this subsection, we compare the EE of RSMA with that of SDMA as SNR increases as well as the corresponding SE and power consumption achieved with both multiple access. As we mentioned in Remark 4, Algorithm 1 can be directly adopted to solve the SE maximization Problem (6) and the EE maximization Problem (8) by setting $w=0$ and $w=1$, respectively.

Fig. 4 shows the EE comparison in different schemes containing 2 users with AoDs $\left[\theta_{1}, \theta_{2}\right]=[0, \pi / 9]$. We 
should notice that when $w=0$, the value of $\mathrm{EE}$ is the ratio of the optimal SE over the power consumption. For the sake of argument, the corresponding denominators and numerators of EE, i.e., achievable SE and achievable transmit power consumption, are also shown in Fig. 5(a) and Fig. 5(b), respectively. Firstly, the EE performance of SCA algorithms and their corresponding Dinkelbach's algorithms are almost the same in both RSMA and SDMA. Furthermore, the proposed low-complexity algorithm, though with a much lower computational complexity, achieves almost the same performance as the SCA algorithm. Secondly, the EE of each algorithm with $w=1$ is equal to that with $w=0$ at low SNR (when $\mathrm{SNR} \leq \mathrm{SNR}_{\text {tradeoff }}=$ $10 \log _{10}\left(\widetilde{p}_{w=1} / \sigma_{k}^{2}\right)$ ), while at high SNR (when SNR > $\left.\mathrm{SNR}_{\text {tradeoff }}=10 \log _{10}\left(\widetilde{p}_{w=1} / \sigma_{k}^{2}\right)\right)$ their behaviors conflict with each other. This is because the aim of Problem (8) is to optimize EE and keep the objective value non-decrease, while that of Problem (6) is to use all the available transmit power to produce maximum SE and even sacrifice EE. Thirdly, the EE produced by RSMA precoder is higher than that generated by SDMA precoder. This behavior is easy to understand in the class of ' $w=0$ ' curves, where RSMA precoder uses the same available power (see Fig. [5(b)) to produce higher achievable SE (see Fig. 5(a)). While in the class of ' $w=1$ ' curves, RSMA precoder uses a little bit higher power (see Fig. 5 b)) to produce much higher SE (see Fig. 5(a)) than SDMA precoder, the resulting EE value of RSMA precoder naturally much higher than that of SDMA precoder. That is to say RSMA shows its crucial benefits in EE communication system. Finally, the behavior of these curves with $w=1$ in Fig. 5(a) and Fig. 5(b) reveals the performance superiority of SCA algorithm over Dinkelbach's algorithm. Basically, while achieving the same high EE, the SE resulted by SCA algorithm is higher than that generated by Dinkelbach's algorithm, which guarantees the QoS of the communication system.s

\section{The SE-EE Tradeoff of the RSMA}

This subsection investigates the SE-EE tradeoff performance of the RSMA and SDMA where the RSMA/SDMA precoders are designed by the LB II since it is more general for $K$-user systems and generates better objective values than the low-complexity algorithm for the two-user system.

Fig. 6 shows the SE-EE tradeoff generated by our proposed weighted-sum approach (10) and weighted-power approach (37) when $K=2$ and $\left[\theta_{1}, \theta_{2}\right]=[0, \pi / 9]$. Firstly, it is obvious that the performances of the weightedsum and weighted-power approaches are the same. While the mathematical model of the weighted-power metric is more concise than the weighted-sum metric. Secondly, when $\mathrm{SNR}=15 \mathrm{~dB}$, the EE and SE remain unchanged in the range of $w$ from 0 to 1 , which indicates that the interests between EE and SE do not conflict with each other at low SNR. Finally, when SNR $=25 \mathrm{~dB}$, we noticed a tradeoff between $\mathrm{EE}$ and SE. Since the performance of the weighted-sum approach and the weighted-power approach is the same, the

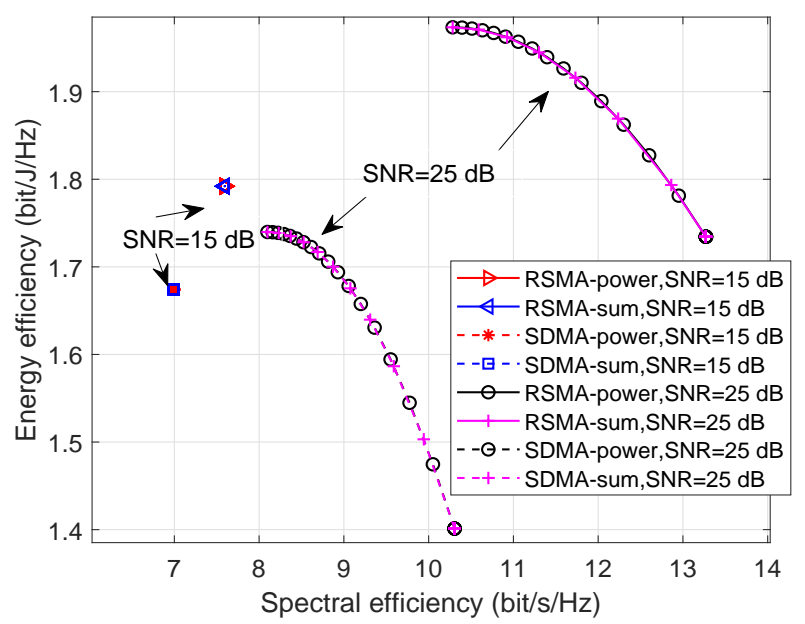

Fig. 6: The SE-EE tradeoff for RSMA/SDMA precoder designed by different approaches with $N_{t}=4, K=2$ and $\chi=0.1 \mathrm{~W} /(\mathrm{bit} / \mathrm{s} / \mathrm{Hz})$.

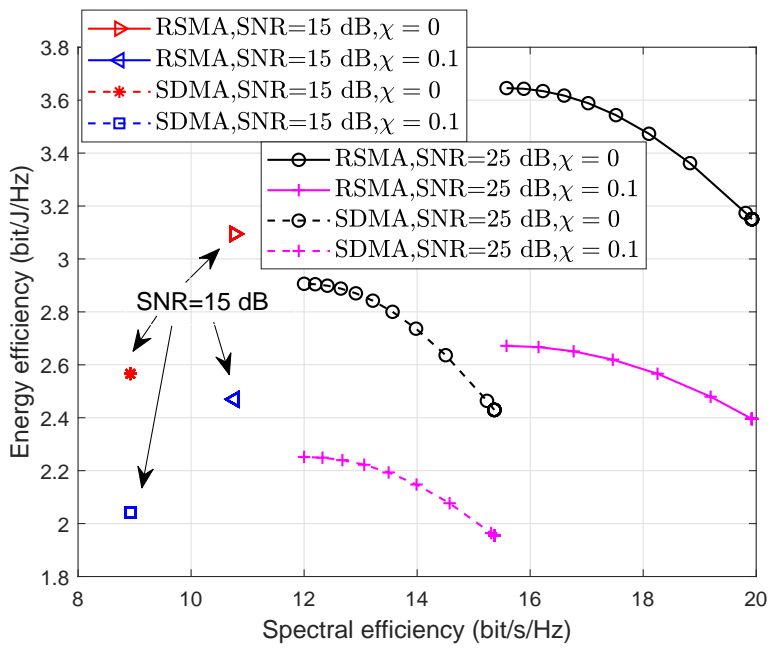

Fig. 7: The SE-EE tradeoff for RSMA/SDMA precoder with $N_{t}=4$ and $K=4$.

following simulations only adopt the weighted-sum approach to investigate the SE-EE tradeoff performance.

Fig. 7 considers the 4 -user case with $\left[\theta_{1}, \theta_{2}, \theta_{3}, \theta_{4}\right]=$ $[0, \pi / 9,2 \pi / 9,3 \pi / 9]$ at different SNR and $\chi$. It is observed that the EE decreases with the increase of $\chi$, but the change of $\chi$ does not affect the SE.

Following the conclusion obtained from Fig. 6 that $\chi$ does not affect the SE, Fig. 8 only depicts the effect of rate-dependent dynamic circuit power consumption on $\mathrm{EE}$ performance when $w=1$. It shows that the increment of $\chi$ leads to the decrease of the EE.

In the following, we introduce another multiple access, namely NOMA [4], as a benchmark. In the considered NOMA scenario of $K$ users, the user with the strongest channel gain needs to decode using SIC the messages of the remaining users before accessing its intended stream. Fig. 9 


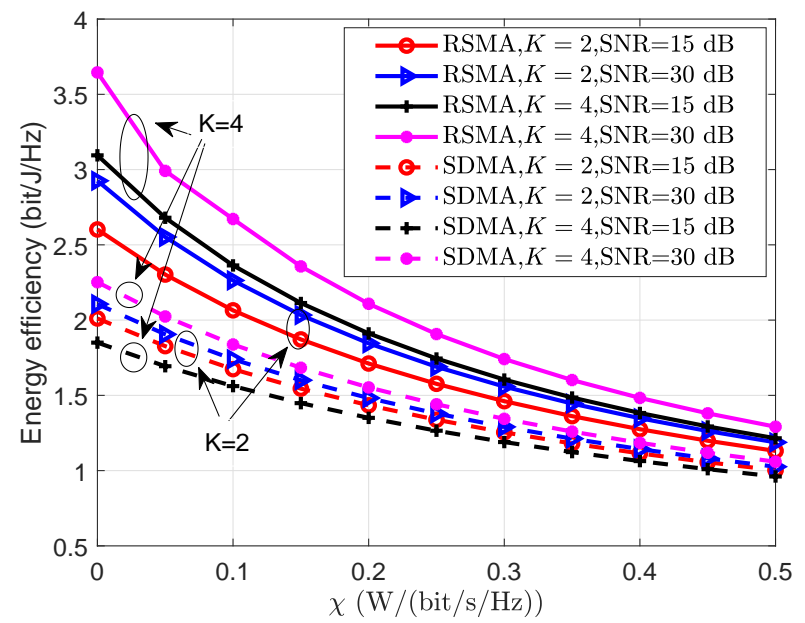

Fig. 8: Energy efficiency versus $\chi$ with $N_{t}=4, K=2$ and $w=1$.

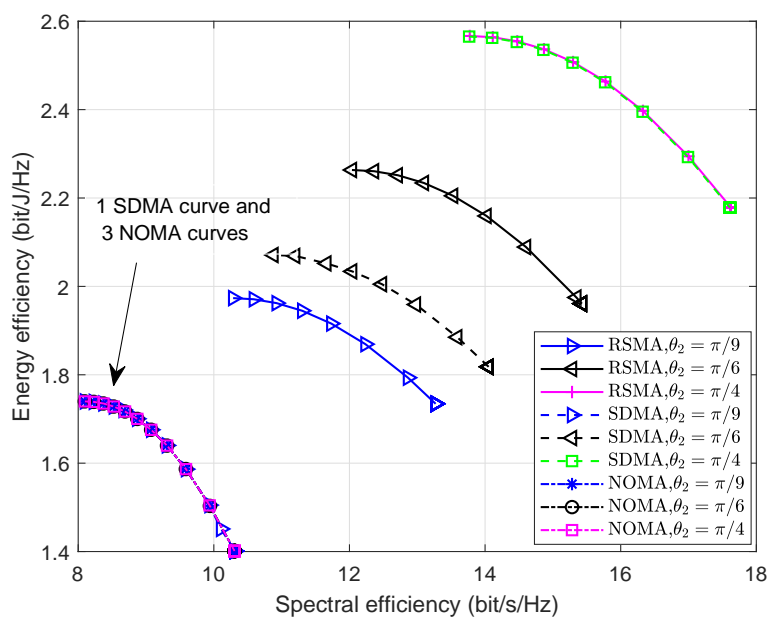

Fig. 9: The SE-EE tradeoff of different AoDs when $\mathrm{SNR}=25$ $\mathrm{dB}, N_{t}=4, K=2$ and $\chi=0.1 \mathrm{~W} /(\mathrm{bit} / \mathrm{s} / \mathrm{Hz})$.

shows the SE-EE tradeoff performance with channel gains $\left\{\nu_{1}=1, \nu_{2}=0.3\right\}$ and different $\theta_{2}=\{\pi / 9, \pi / 6, \pi / 4\}$. It is observed that the AoDs has a significant effect on the performance of the RSMA, but has no effect on that of NOMA. While as for SDMA, when users' channels are aligned (e.g., $\theta_{2}=\{\pi / 9, \pi / 6\}$ ) and the inter-channel interference is large, RSMA can perform more effective interference management than SDMA. When users' channels are nearly orthogonal (e.g., $\theta_{2}=\pi / 4$ ), the interference suppression capability of SDMA is the same as that of RSMA. Generally speaking, the performance of RSMA is better than other multiple access techniques.

Fig. 10 illustrates the effect of $K$ on the SE-EE tradeoff with RSMA, SDMA and NOMA under CSCG random channels. The average trade-off regimes of different multiple access are generated over 200 random channel realizations when $\mathrm{SNR}=25 \mathrm{~dB}$ and $\chi=0.1 \mathrm{~W} /(\mathrm{bit} / \mathrm{s} / \mathrm{Hz})$. It is observed

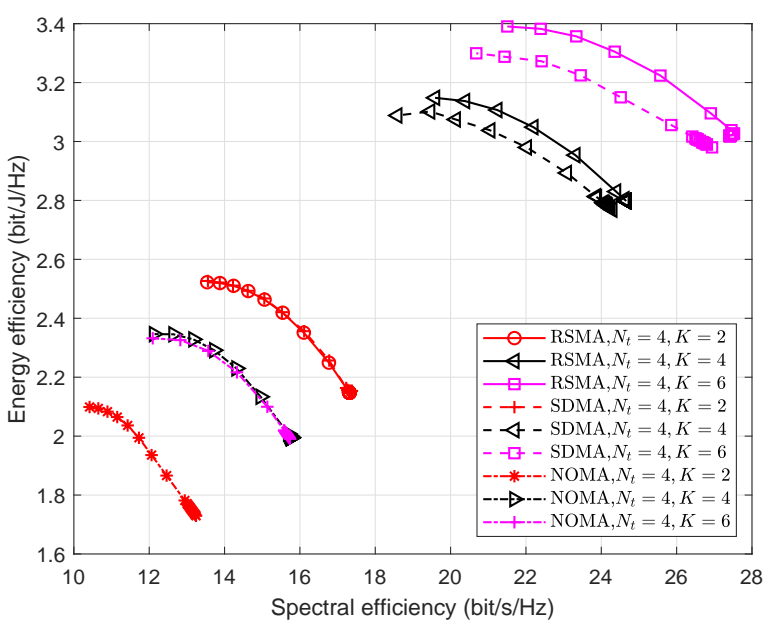

Fig. 10: The SE-EE tradeoff of different users when $S N R=25$ $\mathrm{dB}$ and $\chi=0.1 \mathrm{~W} /(\mathrm{bit} / \mathrm{s} / \mathrm{Hz})$.

that the SE-EE trade-off regime gap between RSMA and SDMA increases as the number of user increases. This shows that the interference management advantage of RSMA over SDMA is more obvious in the overloaded scenario. The performance of NOMA is the worst in this random channel scenario.

\section{CONCLUSION}

In this paper, we have addressed the SE-EE tradeoff of RSMA in a multi-antenna Broadcast Channel and have shown the potential of RSMA to boost the SE-EE tradeoff. The tradeoff problem is a multiple-objective optimization problem and each objective function is non-convex due to the complex sum rate expressions and power consumption expression. In order to overcome those challenges, we firstly proposed two approaches, namely weighted-sum approach and weighted-power approach, to obtain the equivalent single objective optimization problems. Then the closed-form solution of each single objective problem is derived for the two-user system, and a SCA algorithm is used to get the optimal precoder of each single objective problem for the $K$-user system. Numerical results demonstrate that the proposed low-complexity precoding algorithm, though with a much lower computational complexity, achieves almost the same trade-off performance as the proposed SCA suboptimal precoding algorithm. More importantly, compared to the conventional SDMA and NOMA, RSMA has significant performance gains in terms of EE and SE.

\section{APPENDIX A}

PROOF OF LEMMA 1

Proof: We first derive the concave lower bound function (26) of common rate $R_{k}$, and then (28) could be derived following the same procedure directly. For the convenience 
of deduction, (4) needs to be reformulated in a more readable equivalent, as

$$
\begin{aligned}
R_{k} & =\log _{2}\left(1+\frac{\mathbf{h}_{k}^{\mathrm{H}} \mathbf{f}_{k} \mathbf{f}_{k}^{\mathrm{H}} \mathbf{h}_{k}}{\sigma_{k}^{2}+\sum_{i \in \mathcal{K} \backslash\{k\}} \mathbf{h}_{k}^{\mathrm{H}} \mathbf{f}_{i} \mathbf{f}_{i}^{\mathrm{H}} \mathbf{h}_{k}}\right) \\
& =\log _{2}\left(1+I_{-k}^{-1} \mathbf{f}_{k}^{\mathrm{H}} \mathbf{h}_{k} \mathbf{h}_{k}^{\mathrm{H}} \mathbf{f}_{k}\right) \\
& =-\log _{2}\left(1-I_{k}^{-1} \mathbf{f}_{k}^{\mathrm{H}} \mathbf{h}_{k} \mathbf{h}_{k}^{\mathrm{H}} \mathbf{f}_{k}\right)
\end{aligned}
$$

where $I_{-k}=\sigma_{k}^{2}+\sum_{i \in \mathcal{K} \backslash\{k\}} \mathbf{h}_{k}^{\mathrm{H}} \mathbf{f}_{i} \mathbf{f}_{i}^{\mathrm{H}} \mathbf{h}_{k}$ and $I_{k}=$ $I_{-k}+\mathbf{h}_{k}^{\mathrm{H}} \mathbf{f}_{k} \mathbf{f}_{k}^{\mathrm{H}} \mathbf{h}_{k}$. The third equation of (46) follows from applying the Woodbury matrix identity [43]. Let $R_{k}\left(\mathbf{f}_{k}, I_{k}\right)$ represent the third equation of (46) with constraint $I_{k}=\sigma_{k}^{2}+\sum_{i \in \mathcal{K}} \mathbf{h}_{k}^{\mathrm{H}} \mathbf{f}_{i} \mathbf{f}_{i}^{\mathrm{H}} \mathbf{h}_{k},-\log _{2}(\cdot)$ is convex and $1-I_{k}^{-1} \mathbf{f}_{k}^{\mathrm{H}} \mathbf{h}_{k} \mathbf{h}_{k}^{\mathrm{H}} \mathbf{f}_{k}$ is jointly concave in $\left(\mathbf{f}_{k}, I_{k}\right)$, thus $R_{k}\left(\mathbf{f}_{k}, I_{k}\right)$ is jointly convex in $\left(\mathbf{f}_{k}, I_{k}\right)$ [40] and minorized by its first-order approximation at fixed point $\left(\mathbf{f}_{k}^{(n)}, I_{k}^{(n)}\right)$. Specifically,

$$
\begin{aligned}
R_{k}\left(\mathbf{f}_{k}, I_{k}\right) \\
\geq R_{k}\left(\mathbf{f}_{k}^{(n)}, I_{k}^{(n)}\right)+\left(\left.\frac{\partial R_{k}}{\partial \mathbf{f}_{k}}\right|_{\mathbf{f}_{k}=\mathbf{f}_{k}^{(n)}}\right)^{\mathrm{T}}\left(\mathbf{f}_{k}-\mathbf{f}_{k}^{(n)}\right) \\
\quad+\left(\left.\frac{\partial R_{k}}{\partial \mathbf{f}_{k}^{*}}\right|_{\mathbf{f}_{k}^{*}=\mathbf{f}_{k}^{(n), *}}\right)^{\mathrm{T}}\left(\mathbf{f}_{k}^{*}-\mathbf{f}_{k}^{(n), *}\right) \\
\quad+\left.\frac{\partial R_{k}}{\partial I_{k}}\right|_{I_{k}=I_{k}^{(n)}}\left(I_{k}-I_{k}^{(n)}\right) \\
=R_{k}\left(\mathbf{f}_{k}^{(n)}, I_{k}^{(n)}\right)+2 \operatorname{Re}\left\{a_{k} \mathbf{b}_{k}^{\mathrm{H}}\left(\mathbf{f}_{k}-\mathbf{f}_{k}^{(n)}\right)\right\} \\
\quad-a_{k}\left(I_{k}^{(n)}\right)^{-2} \mathbf{f}_{k}^{(n), \mathrm{H}} \mathbf{h}_{k} \mathbf{h}_{k}^{\mathrm{H}} \mathbf{f}_{k}^{(n)}\left(I_{k}-I_{k}^{(n)}\right),
\end{aligned}
$$

where $a_{k}$ and $\mathbf{b}_{k}$ are defined in (27a) and 27b), respectively. Undo $I_{k}=\sigma_{k}^{2}+\sum_{i \in \mathcal{K}} \mathbf{h}_{k}^{\mathrm{H}} \mathbf{f}_{i} \mathbf{f}_{i}^{\mathrm{H}} \mathbf{h}_{k}$ and $I_{k}^{n}=\sigma_{k}^{2}+$ $\sum_{i \in \mathcal{K}} \mathbf{h}_{k}^{\mathrm{H}} \mathbf{f}_{i}^{(n)} \mathbf{f}_{i}^{(n), \mathrm{H}} \mathbf{h}_{k}$, the last equation of (47) equals (26).

Hence, the proof is completed.

\section{APPENDIX B}

\section{Proof OF Proposition 1}

In the following, we prove that $\mathbf{F}^{o}$ is the KKT point based on the fact that all the globally optimal solutions of a convex optimization problem should satisfy the KKT optimality conditions [40].

Since (30) and (34) are both convex problems derived from SCA method, the optimal solutions of these two problems have the same property. Here, we only prove in detail that the optimal solution of Problem (30) is the KKT optimality for Problem (10).

Firstly, denote by $\left\{\mathbf{F}^{o}, \eta^{o}, \mathbf{r}^{o}, x^{o}, y^{o}\right\}$ the converged solutions of Problem (30) whose Lagrangian is then given by

$$
\begin{aligned}
& \mathcal{L}(\mathbf{F}, \eta, \mathbf{r}, x, y, \boldsymbol{\lambda}) \\
= & w \eta+\frac{(1-w)}{\mathrm{P}_{\mathrm{c}}} \sum_{i \in \mathcal{K}_{\mathrm{c}}} r_{i}-\sum_{k \in \mathcal{K}} \lambda_{k}^{(1)}\left(r_{k}-R_{k}^{(n)}\left(\mathbf{F} \mid \mathbf{F}^{o}\right)\right) \\
& -\sum_{k \in \mathcal{K}} \lambda_{k}^{(2)}\left(r_{\mathrm{c}}-R_{\mathrm{c}, k}^{(n)}\left(\mathbf{F} \mid \mathbf{F}^{o}\right)\right)-\lambda^{(3)}\left(\eta-\phi^{(n)}\left(x, y \mid x^{o}, y^{o}\right)\right) \\
& -\lambda^{(4)}\left(x^{2}-\sum_{i \in \mathcal{K}_{\mathrm{c}}} r_{i}\right)-\lambda^{(5)}\left(\|\mathbf{F}\|_{F}^{2}+\mathrm{P}_{\mathrm{c}}+\chi \sum_{i \in \mathcal{K}_{\mathrm{c}}} r_{i}-y\right),
\end{aligned}
$$

where $\boldsymbol{\lambda}=\left[\lambda_{1}^{(1)}, \ldots, \lambda_{K}^{(1)}, \lambda_{1}^{(2)}, \ldots, \lambda_{K}^{(2)}, \lambda^{(3)}, \lambda^{(4)}, \lambda^{(5)}\right]^{\mathrm{T}}$ are the dual variables. There must exist a $\lambda^{o}$ satisfying the following partial KKT conditions:

$$
\begin{aligned}
& \nabla_{\mathbf{F}^{*}} \mathcal{L}=\left.\sum_{k \in \mathcal{K}} \lambda_{k}^{(1), o} \nabla_{\mathbf{F}^{*}} R_{k}^{(n)}\left(\mathbf{F} \mid \mathbf{F}^{o}\right)\right|_{\mathbf{F}=\mathbf{F}^{o}} \\
& +\left.\sum_{k \in \mathcal{K}} \lambda_{k}^{(2), o} \nabla_{\mathbf{F}^{*}} R_{\mathrm{c}, k}^{(n)}\left(\mathbf{F} \mid \mathbf{F}^{o}\right)\right|_{\mathbf{F}=\mathbf{F}^{o}-\lambda^{(5), o} \mathbf{F}^{o}=\mathbf{0}}
\end{aligned}
$$$$
\nabla_{x} \mathcal{L}=\lambda^{(3), o} \frac{2 x^{o}}{y^{o}}-2 \lambda^{(4), o} x^{o}=0,
$$

$$
\nabla_{y} \mathcal{L}=\lambda^{(5), o}-\lambda^{(3), o}\left(\frac{x^{o}}{y^{o}}\right)^{2}=0,
$$

$\lambda_{k}^{(1), o}\left(r_{k}^{o}-R_{k}^{(n)}\left(\mathbf{F}^{o} \mid \mathbf{F}^{o}\right)\right)=0, \forall k \in \mathcal{K}$,

$\lambda_{k}^{(2), o}\left(r_{\mathrm{c}}^{o}-R_{\mathrm{c}, k}^{(n)}\left(\mathbf{F}^{o} \mid \mathbf{F}^{o}\right)\right)=0, \forall k \in \mathcal{K}$,

$\lambda^{(3), o}\left(\eta-\phi^{(n)}\left(x^{o}, y^{o}\right)\right)=0$,

$$
\lambda^{(5), o}\left(\left\|\mathbf{F}^{o}\right\|_{F}^{2}+\mathrm{P}_{\mathrm{c}}+\chi \sum_{i \in \mathcal{K}_{\mathrm{c}}} r_{i}^{o}-y^{o}\right)=0 .
$$

It is straightforward that

$$
\begin{aligned}
& R_{k}^{(n)}\left(\mathbf{F}^{o} \mid \mathbf{F}^{o}\right)=R_{k}\left(\mathbf{F}^{o}\right), \\
& R_{\mathrm{c}, k}^{(n)}\left(\mathbf{F}^{o} \mid \mathbf{F}^{o}\right)=R_{\mathrm{c}, k}\left(\mathbf{F}^{o}\right) .
\end{aligned}
$$

Furthermore, the first-order partial derivatives with respect to $\mathbf{F}$ for $R_{k}(\mathbf{F})$ and $R_{k}^{(n)}\left(\mathbf{F} \mid \mathbf{F}^{o}\right)$ are given by

$$
\left.\nabla_{\mathbf{f}_{k}^{*}} R_{k}(\mathbf{F})\right|_{\mathbf{f}_{k}^{*}=\mathbf{f}_{k}^{*, o}}=\frac{\mathbf{h}_{k}^{\mathrm{H}} \mathbf{f}_{k}^{o} \mathbf{h}_{k}}{I_{k}^{o}}=\left.\nabla_{\mathbf{f}_{k}^{*}} R_{k}^{(n)}\left(\mathbf{F} \mid \mathbf{F}^{o}\right)\right|_{\mathbf{f}_{k}^{*}=\mathbf{f}_{k}^{*, o}},
$$

$$
\begin{aligned}
\left.\nabla_{\mathbf{f}_{i}^{*}} R_{k}(\mathbf{F})\right|_{\mathbf{f}_{i}^{*}=\mathbf{f}_{i}^{*, o}} & =-\frac{\mathbf{h}_{k}^{\mathrm{H}} \mathbf{f}_{k}^{o} \mathbf{f}_{k}^{\mathrm{H}, o} \mathbf{h}_{k} \mathbf{h}_{k}^{\mathrm{H}} \mathbf{f}_{i}^{o} \mathbf{h}_{k}}{I_{k}^{o} I_{-k}^{o}} \\
& =\left.\nabla_{\mathbf{f}_{i}^{*}} R_{k}^{(n)}\left(\mathbf{F} \mid \mathbf{F}^{o}\right)\right|_{\mathbf{f}_{i}^{*}=\mathbf{f}_{i}^{*}, o},
\end{aligned}
$$

where $I_{-k}^{o}=\sigma_{k}^{2}+\sum_{i \in \mathcal{K} \backslash\{k\}} \mathbf{h}_{k}^{\mathrm{H}} \mathbf{f}_{i}^{o} \mathbf{f}_{i}^{\mathrm{H}, o} \mathbf{h}_{k}$ and $I_{k}^{o}=I_{-k}^{o}+$ $\mathbf{h}_{k}^{\mathrm{H}} \mathbf{f}_{k}^{o} \mathbf{f}_{k}^{\mathrm{H}, o} \mathbf{h}_{k}$. With (50), we arrive at

$$
\left.\nabla_{\mathbf{F}} R_{k}(\mathbf{F})\right|_{\mathbf{F}=\mathbf{F}^{o}}=\left.\nabla_{\mathbf{F}} R_{k}^{(n)}\left(\mathbf{F} \mid \mathbf{F}^{o}\right)\right|_{\mathbf{F}=\mathbf{F}^{o}} .
$$

Then, the equivalence relation between two first-order partial derivatives of $R_{\mathrm{c}, k}(\mathbf{F})$ and $R_{\mathrm{c}, k}^{(n)}\left(\mathbf{F} \mid \mathbf{F}^{o}\right)$ is directly given by

$$
\left.\nabla_{\mathbf{F}} R_{\mathrm{c}, k}(\mathbf{F})\right|_{\mathbf{F}=\mathbf{F}^{o}}=\left.\nabla_{\mathbf{F}} R_{\mathrm{c}, k}^{(n)}\left(\mathbf{F} \mid \mathbf{F}^{o}\right)\right|_{\mathbf{F}=\mathbf{F}^{o}} .
$$

By substituting (51) and (52) into 448a), and substituting (49a) and (49b) into (48d) and (48e) respectively, we obtain

$$
\begin{aligned}
& \left.\sum_{k \in \mathcal{K}} \lambda_{k}^{(1), o} \nabla_{\mathbf{F}^{*}} R_{k}(\mathbf{F})\right|_{\mathbf{F}=\mathbf{F}^{o}} \\
& +\left.\sum_{k \in \mathcal{K}} \lambda_{k}^{(2), o} \nabla_{\mathbf{F}^{*}} R_{\mathrm{c}, k}(\mathbf{F})\right|_{\mathbf{F}=\mathbf{F}^{o}-\lambda^{(5), o} \mathbf{F}^{o}=\mathbf{0},} \\
& \lambda_{k}^{(1), o}\left(r_{k}^{o}-R_{k}\left(\mathbf{F}^{o}\right)\right)=0, \forall k \in \mathcal{K}, \\
& \lambda_{k}^{(2), o}\left(r_{\mathrm{c}}^{o}-R_{\mathrm{c}, k}\left(\mathbf{F}^{o}\right)\right)=0, \forall k \in \mathcal{K}
\end{aligned}
$$

Now, it can be readily verified that the set of equations 48b), 48c), 48f, 48g, and (53) constitute exactly the 
KKT conditions of Problem (24). Since Problem (24) is equivalent to Problem (10), $\mathbf{F}^{o}$ is the KKT optimality for Problem (10).

Hence, the proof is completed.

\section{REFERENCES}

[1] Te Han and K. Kobayashi, "A new achievable rate region for the interference channel," IEEE Trans. Inf. Theory, vol. 27, no. 1, pp. 49-60, Jan. 1981.

[2] B. Clerckx, H. Joudeh, C. Hao, M. Dai, and B. Rassouli, "Rate splitting for MIMO wireless networks: A promising PHY-layer strategy for LTE evolution," IEEE Commun. Mag., vol. 54, no. 5, pp. 98-105, May 2016.

[3] B. Clerckx, Y. Mao, R. Schober, and H. V. Poor, "Rate-splitting unifying SDMA, OMA, NOMA, and multicasting in MISO broadcast channel: A simple two-user rate analysis," IEEE Signal Process. Lett., vol. 9, no. 3, pp. 349-353, 2020.

[4] Y. Mao, B. Clerckx, and V. O. K. Li, "Rate-splitting for multiantenna non-orthogonal unicast and multicast transmission: Spectral and energy efficiency analysis," IEEE Trans. Commun., pp. 1-1, Sep. 2019.

[5] Y. Mao, B. Clerckx, and V. O. Li, "Rate-splitting multiple access for downlink communication systems: Bridging, generalizing, and outperforming SDMA and NOMA," EURASIP J. Wireless Commun. Netw., vol. 2018, no. 1, p. 133, May 2018.

[6] A. Alameer Ahmad, H. Dahrouj, A. Chaaban, A. Sezgin, and M. Alouini, "Interference mitigation via rate-splitting and common message decoding in cloud radio access networks," IEEE Access, vol. 7, pp. 80350-80365, Jun. 2019.

[7] Y. Mao, B. Clerckx, and V. O. K. Li, "Rate-splitting multiple access for coordinated multi-point joint transmission," in Proc. IEEE Int Conf. Commun. (ICC Workshops), May 2019, pp. 1-6.

[8] C. Hao, Y. Wu, and B. Clerckx, "Rate analysis of two-receiver MISO broadcast channel with finite rate feedback: A rate-splitting approach," IEEE Trans. Commun., vol. 63, no. 9, pp. 3232-3246, Sep. 2015.

[9] M. Dai, B. Clerckx, D. Gesbert, and G. Caire, "A rate splitting strategy for massive MIMO with imperfect CSIT," IEEE Trans. Wireless Commun., vol. 15, no. 7, pp. 4611-4624, Jul. 2016.

[10] M. Dai and B. Clerckx, "Multiuser millimeter wave beamforming strategies with quantized and statistical CSIT," IEEE Trans. Wireless Commun., vol. 16, no. 11, pp. 7025-7038, Nov. 2017.

[11] H. Joudeh and B. Clerckx, "Robust transmission in downlink multiuser MISO systems: A rate-splitting approach," IEEE Trans. Signal Process., vol. 64, no. 23, pp. 6227-6242, Dec. 2016.

[12] _ - "Sum-rate maximization for linearly precoded downlink multiuser MISO systems with partial CSIT: A rate-splitting approach," IEEE Trans. Commun., vol. 64, no. 11, pp. 4847-4861, Nov. 2016.

[13] _ - "Rate-splitting for max-min fair multigroup multicast beamforming in overloaded systems," IEEE Trans. Wireless Commun., vol. 16, no. 11, pp. 7276-7289, Nov. 2017.

[14] Y. Mao and B. Clerckx, "Beyond dirty paper coding for multi-antenna broadcast channel with partial csit: A rate-splitting approach," IEEE Trans. Commun., early access.

[15] J. Zhang, B. Clerckx, J. Ge, and Y. Mao, "Cooperative rate-splitting for MISO broadcast channel with user relaying, and performance benefits over cooperative NOMA," IEEE Signal Process. Lett., vol. 26, no. 11 , pp. $1678-1682,2019$.

[16] Y. Mao, B. Clerckx, J. Zhang, V. O. K. Li, and M. Arafah, "Maxmin fairness of K-user cooperative rate-splitting in MISO broadcast channel with user relaying," IEEE Trans. Wireless Commun., early access.

[17] Y. Mao, B. Clerckx, and V. O. K. Li, "Energy efficiency of ratesplitting multiple access, and performance benefits over SDMA and NOMA," Proc. IEEE Int. Symp. Wireless Commun. Syst. (ISWCS), 2018.

[18] Y. Chen, S. Zhang, S. Xu, and G. Y. Li, "Fundamental trade-offs on green wireless networks," IEEE Commun. Mag., vol. 49, no. 6, pp. 30-37, June 2011.

[19] S. He, Y. Huang, S. Jin, F. Yu, and L. Yang, "Max-min Energy efficient beamforming for multicell multiuser joint transmission systems," IEEE Commun. Lett., vol. 17, no. 10, pp. 1956-1959, Oct. 2013.
[20] C. Xiong, G. Y. Li, S. Zhang, Y. Chen, and S. Xu, "Energy-and spectral-efficiency tradeoff in downlink OFDMA networks," IEEE Trans. Wireless Commun., vol. 10, no. 11, pp. 3874-3886, Nov. 2011.

[21] O. Amin, E. Bedeer, M. H. Ahmed, and O. A. Dobre, "Energy efficiency-spectral efficiency tradeoff: A multiobjective optimization approach," IEEE Trans. Veh. Technol., vol. 65, no. 4, pp. 1975-1981, Apr. 2016.

[22] R. Zhang, Y. Li, C. Wang, Y. Ruan, Y. Fu, and H. Zhang, "Energyspectral efficiency trade-off in underlaying mobile D2D communications: An economic efficiency perspective," IEEE Trans. Wireless Commun., vol. 17, no. 7, pp. 4288-4301, Jul. 2018.

[23] O. Tervo, L. Tran, H. Pennanen, S. Chatzinotas, B. Ottersten, and M. Juntti, "Energy-efficient multicell multigroup multicasting with joint beamforming and antenna selection," IEEE Trans. Signal Process., vol. 66, no. 18, pp. 4904-4919, Sep. 2018.

[24] W. Dinkelbach, "On nonlinear fractional programming," Manage. Sci., vol. 13, no. 7, pp. 492-498, 1967.

[25] M. Razaviyayn, Successive convex approximation: Analysis and applications. Ph.D. dissertation, Univ. Minnesota, Minneapolis, MN, USA, 2014.

[26] O. Tervo, L. Tran, and M. Juntti, "Optimal energy-efficient transmit beamforming for multi-user MISO downlink," IEEE Trans. Signal Process., vol. 63, pp. 5574-5588, Jul. 2015.

[27] K. Nguyen, Q. Vu, L. Tran, and M. Juntti, "Energy efficiency fairness for multi-pair wireless-powered relaying systems," IEEE J. Select. Areas Commun., vol. 37, no. 2, pp. 357-373, Feb. 2019.

[28] G. Caire and S. Shamai, "On the achievable throughput of a multiantenna Gaussian broadcast channel," IEEE Trans. Inform. Theory, vol. 49, no. 7, pp. 1691-1706, Jul. 2003.

[29] S. S. Christensen, R. Agarwal, E. D. Carvalho, and J. M. Cioffi, "Weighted sum-rate maximization using weighted MMSE for MIMOBC beamforming design," IEEE Trans. Wireless Commun., vol. 7, no. 12, pp. 4792-4799, Dec. 2008.

[30] C. Pan, W. Xu, J. Wang, H. Ren, W. Zhang, N. Huang, and M. Chen, "Pricing-based distributed energy-efficient beamforming for MISO interference channels," IEEE J. Select. Areas Commun., vol. 34, no. 4, pp. 710-722, Apr. 2016.

[31] A. Zappone, E. Björnson, L. Sanguinetti, and E. A. Jorswieck, "Globally optimal energy-efficient power control and receiver design in wireless networks," IEEE Trans. Signal Process., vol. 65, no. 11, Jun. 2017.

[32] B. Matthiesen, C. Hellings, and E. A. Jorswieck, "Energy efficiency: Rate splitting vs. point-to-point codes in gaussian interference channels," in Proc. SPAWC, Jul. 2019, pp. 1-5.

[33] E. Björnson, L. Sanguinetti, J. Hoydis, and M. Debbah, "Optimal design of energy-efficient multi-user MIMO systems: Is massive MIMO the answer?" IEEE Trans. Wireless Commun., vol. 14, no. 6, pp. 3059-3075, Jun. 2015.

[34] K. Xiong, P. Fan, Y. Lu, and K. B. Letaief, "Energy efficiency with proportional rate fairness in multirelay OFDM networks," IEEE $J$. Select. Areas Commun., vol. 34, no. 5, pp. 1431-1447, May 2016.

[35] C. Isheden and G. P. Fettweis, "Energy-efficient multi-carrier link adaptation with sum rate-dependent circuit power," in Proc. IEEE Glob. Commun. Conf. (GLOBECOM), Dec. 2010, pp. 1-6.

[36] M. Ehrgott, Multicriteria Optimization. New York, NY, USA: Springer-Verlag, 2005.

[37] B. Clerckx, Y. Mao, R. Schober, and H. V. Poor, "Rate-splitting unifying SDMA, OMA, NOMA, and multicasting in MISO broadcast channel: A simple two-user rate analysis," IEEE Wireless Commun. Lett., vol. 9, no. 3, pp. 349-353, Mar. 2020.

[38] C. Hsiao, j.C. Guey, W. Sheen, and R. Chen, "A two-user approximation-based transmit beamforming for physical-layer multicasting in mobile cellular downlink systems," J. Chin. Inst. Eng., vol. 38, no. 6, pp. 742-750, 2015.

[39] Y. Yang, M. Pesavento, S. Chatzinotas, and B. Ottersten, "Energy efficiency optimization in MIMO interference channels: A successive pseudoconvex approximation approach," IEEE Trans. Signal Process., vol. 67 , no. 15 , pp. 4107-4121, Aug. 2019.

[40] S. Boyd and L. Vandenberghe, Convex optimization. Cambridge Univ. Press, 2004.

[41] B. R. Marks and G. P. Wright, "A general inner approximation algorithm for nonconvex mathematical programs," Oper. Res., vol. 26, no. 4, pp. 681-683, Jul.-Aug. 1978.

[42] "The mosek optimization toolbox for MATLAB manual," Version 7.1 (revision 28). [Online] http://mosek.com, accessed on: Mar. 20, 2015. 
[43] N. Higham, Accuracy and stability of numerical algorithms (2nd ed.). SIAM, 2002. 
This figure "EE-SNR.jpg" is available in "jpg" format from: http://arxiv.org/ps/2001.03206v2 
This figure "auther-Gui-Zhou.jpg" is available in "jpg" format from: http://arxiv.org/ps/2001.03206v2 
This figure "bullet.png" is available in "png" format from: http://arxiv.org/ps/2001.03206v2 
This figure "fig1.png" is available in "png" format from: http://arxiv.org/ps/2001.03206v2 
This figure "logo.png" is available in "png" format from: http://arxiv.org/ps/2001.03206v2 
This figure "notaglinelogo.png" is available in "png" format from: http://arxiv.org/ps/2001.03206v2 